\title{
Other articles noted 25 Jul 03 to 7 Nov 03
}

A list of the journals that are reviewed and the criteria for selecting articles from these journals for inclusion in EvidenceBased Nursing are included in each issue. The following articles passed all criteria but were not abstracted because, in the judgment of the editors, their findings were less widely applicable to nursing practice, the topic was of interest to only a select group of nurse specialists, or the topic was recently addressed in another abstract.

\section{TREATMENT}

A randomized controlled study of the acute and chronic effects of cooling therapy for MS. Neurology 2003;60:1955-60. http://www.ncbi.nlm.nih.gov/entrez/ query.fcgi?cmd $=$ Retrieve\&db $=$ PubMed\&list_uids $=12821739 \& \mathrm{dopt}=$ Abstract

Aaron SD, Vandemheen KL, Hebert P, et al. Outpatient oral prednisone after emergency treatment of chronic obstructive pulmonary disease. $N$ Engl J Med 2003;348:2618-25. http://www.ncbi.nlm.nih.gov/entrez/ query.fcgi?cmd $=$ Retrieve\&db $=$ PubMed\&list_uids $=12826636 \& \mathrm{dopt}=$ Abstract

Agnelli G, Prandoni P, Becattini C, et al. Extended oral anticoagulant therapy after a first episode of pulmonary embolism. Ann Intern Med 2003;139:19-25.

http://www.ncbi.nlm.nih.gov/entrez/

query.fcgi?cmd $=$ Retrieve\&db $=$ PubMed\&list_uids

$=12834314 \& \mathrm{dopt}=$ Abstract

Akhondzadeh S, Noroozian M, Mohammadi M, et al. Melissa officinalis extract in the treatment of patients with mild to moderate Alzheimer's disease: a double blind, randomised, placebo controlled trial. $J$ Neurol Neurosurg Psychiatry 2003;74:863-6.

http://www.ncbi.nlm.nih.gov/entrez/

query.fcgi?cmd $=$ Retrieve $\& \mathrm{db}=$ PubMed\&list_uids

$=12810768 \& \mathrm{dopt}=$ Abstract

Anderson GL, Judd HL, Kaunitz AM, et al. Effects of estrogen plus progestin on gynecologic cancers and associated diagnostic procedures: the Women's Health Initiative randomized trial. JAMA 2003;290:1739-48.

http://www.ncbi.nlm.nih.gov/entrez/

query.fcgi?cmd $=$ Retrieve\&db = PubMed\&list_uids

$=14519708 \& \mathrm{dopt}=$ Abstract

Arshad SH, Bateman B, Matthews SM. Primary prevention of asthma and atopy during childhood by allergen avoidance in infancy: a randomised controlled study. Thorax 2003;58:48993.

http://www.ncbi.nlm.nih.gov/entrez/

query.fcgi?cmd $=$ Retrieve\&db $=$ PubMed\&list_uids

$=12775858 \& \mathrm{dopt}=$ Abstract

Ambalavanan N, Wu TJ, Tyson JE, et al. A comparison of three vitamin A dosing regimens in extremely-low-birthweight infants. J Pediatr 2003;142:656-61.

http://www.ncbi.nlm.nih.gov/entrez/

query.fcgi?cmd $=$ Retrieve\&db $=$ PubMed\&list_uids

$=12838194 \& \mathrm{dopt}=$ Abstract

Amirav I, Balanov I, Gorenberg M, et al. Nebuliser hood compared to mask in wheezy infants: aerosol therapy without tears! Arch Dis Child 2003;88:719-23. http://www.ncbi.nlm.nih.gov/entrez/

query.fcgi?cmd $=$ Retrieve $\& \mathrm{db}=$ PubMed\&list_uids

$=12876173 \& \mathrm{dopt}=$ Abstract

Barnett A, Smith B, Lord SR, et al. Community-based group exercise improves balance and reduces falls in at-risk older people: a randomised controlled trial. Age Ageing 2003;32: 407-14.

http://www.ncbi.nlm.nih.gov/entrez/

query.fcgi?cmd = Retrieve\&db = PubMed\&list_uids

$=12851185 \& \mathrm{dopt}=$ Abstract

Beardslee WR, Gladstone TR, Wright EJ, et al. A family-based approach to the prevention of depressive symptoms in children at risk: evidence of parental and child change. Pediatrics 2003;112:el19-31.

http://www.ncbi.nlm.nih.gov/entrez/

query.fcgi?cmd $=$ Retrieve $\& \mathrm{db}=$ PubMed\&list_uids

$=12897317 \&$ dopt $=$ Abstract

Beich A, Thorsen T, Rollnick S. Screening in brief intervention trials targeting excessive drinkers in general practice: systematic review and meta-analysis. BMJ 2003;327:536-42. http://www.ncbi.nlm.nih.gov/entrez/

query.fcgi?cmd $=$ Retrieve $\& \mathrm{db}=$ PubMed\&list_uids

$=12958114 \& \mathrm{dopt}=$ Abstract

Bekker HL, Legare F, Stacey D, et al. Is anxiety a suitable measure of decision aid effectiveness: a systematic review? Patient Educ Couns 2003;50:255-62.

http://www.ncbi.nlm.nih.gov/entrez/

query.fcgi?cmd $=$ Retrieve $\& d b=$ PubMed\&list_uids

$=12900095 \& \mathrm{dopt}=$ Abstract

Berkman LF, Blumenthal J, Burg M, et al. Effects of treating depression and low perceived social support on clinical events after myocardial infarction: the Enhancing Recovery in Coronary Heart Disease Patients (ENRICHD) Randomized Trial. JAMA 2003;289:3106-16.

http://www.ncbi.nlm.nih.gov/entrez/

query.fcgi?cmd $=$ Retrieve $\& \mathrm{db}=$ PubMed\&list_uids

$=12813116 \&$ dopt $=$ Abstract

Blackhall K, Appleton S, Cates CJ. Ionisers for chronic asthma. Cochrane Database Syst Rev 2003;(3):CD002986.

http://www.ncbi.nlm.nih.gov/entrez/

query.fcgi?cmd $=$ Retrieve $\& \mathrm{db}=$ PubMed\&list_uids

$=12917939 \& \mathrm{dopt}=$ Abstract

Bloomfield P, Hodson EM, Craig JC. Antibiotics for acute pyelonephritis in children. Cochrane Database Syst Rev 2003; (3):CD003772.

http://www.ncbi.nlm.nih.gov/entrez/

query.fcgi?cmd $=$ Retrieve $\& \mathrm{db}=$ PubMed\&list_uids

$=12917987 \& \mathrm{dopt}=$ Abstract

Bong JJ, Kite $\mathrm{P}$, Wilco $\mathrm{MH}$, et al. Prevention of catheter related bloodstream infection by silver iontophoretic central 
2 of 14

venous catheters: a randomised controlled trial. J Clin Pathol 2003;56:731-5.

http://www.ncbi.nlm.nih.gov/entrez/

query.fcgi? $\mathrm{cmd}=$ Retrieve $\& \mathrm{db}=$ PubMed\&list_uids

$=14514774 \& \mathrm{dopt}=$ Abstract

Borghi C, Bacchelli S, Esposti DD, et al. Effects of the early ACE inhibition in diabetic nonthrombolyzed patients with anterior acute myocardial infarction. Diabetes Care 2003;26:1862-8. http://www.ncbi.nlm.nih.gov/entrez/ query.fcgi?cmd $=$ Retrieve $\& \mathrm{db}=$ PubMed\&list_uids $=12766124 \& \mathrm{dopt}=$ Abstract

Bott U, Ebrahim S, Hirschberger S, et al. Effect of the rapidacting insulin analogue insulin aspart on quality of life and treatment satisfaction in patients with Type 1 diabetes. Diabet Med 2003;20:626-34.

http://www.ncbi.nlm.nih.gov/entrez/

query.fcgi?cmd $=$ Retrieve $\& \mathrm{db}=$ PubMed\&list_uids

$=12873289 \& \mathrm{dopt}=$ Abstract

Brand-Miller J, Hayne S, Petocz P, et al. Low-glycemic index diets in the management of diabetes: a meta-analysis of randomized controlled trials. Diabetes Care 2003;26:2261-7.

http://www.ncbi.nlm.nih.gov/entrez/

query.fcgi?cmd $=$ Retrieve $\& \mathrm{db}=$ PubMed\&list_uids

$=12882846 \& \mathrm{dopt}=$ Abstract

Brosseau L, Yonge KA, Robinson V, et al. Transcutaneous electrical nerve stimulation (TENS) for the treatment of rheumatoid arthritis in the hand. Cochrane Database Syst Rev 2003;(3):CD004287.

http://www.ncbi.nlm.nih.gov/entrez/

query.fcgi?cmd $=$ Retrieve $\& \mathrm{db}=$ PubMed\&list_uids

$=12918009 \& \mathrm{dopt}=$ Abstract

Bucher HC, Tschudi P, Young J, et al. Effect of amoxicillinclavulanate in clinically diagnosed acute rhinosinusitis: a placebo-controlled, double-blind, randomized trial in general practice. Arch Intern Med 2003;163:1793-8.

http://www.ncbi.nlm.nih.gov/entrez/

query.fcgi?cmd $=$ Retrieve $\& \mathrm{db}=$ PubMed\&list_uids

$=12912714 \& \mathrm{dopt}=$ Abstract

Cameron M, Lonergan E, Lee H. Transcutaneous electrical nerve stimulation (TENS) for dementia. Cochrane Database Syst Rev 2003;(3):CD004032.

http://www.ncbi.nlm.nih.gov/entrez/

query.fcgi?cmd $=$ Retrieve $\& \mathrm{db}=$ PubMed\&list_uids

$=12917999 \& \mathrm{dopt}=$ Abstract

Carfoot S, Williamson PR, Dickson R. A systematic review of randomised controlled trials evaluating the effect of mother/ baby skin-to-skin care on successful breast feeding. Midwifery 2003;19:148-55.

http://www.ncbi.nlm.nih.gov/entrez/

query.fcgi cmd $=$ Retrieve $\& \mathrm{db}=$ PubMed\&list_uids

$=12809635 \& \mathrm{dopt}=$ Abstract

Chen YY, Yen DH, Yang YG, et al. Comparison between replacement at 4 days and 7 days of the infection rate for pulmonary artery catheters in an intensive care unit. Crit Care Med 2003:31:1353-8.

http://www.ncbi.nlm.nih.gov/entrez/

query.fcgi? $\mathrm{cmd}=$ Retrieve $\& \mathrm{db}=$ PubMed\&list_uids $=12771602 \& \mathrm{dopt}=$ Abstract

Cheyne H, Dunlop A, Shields N, et al. A randomised controlled trial of admission electronic fetal monitoring in normal labour. Midwifery 2003;19:221-9. http://www.ncbi.nlm.nih.gov/entrez/

query.fcgi?cmd $=$ Retrieve $\& \mathrm{db}=$ PubMed\&list_uids

$=12946338 \& \mathrm{dopt}=$ Abstract

Cimprich B, Ronis DL. An environmental intervention to restore attention in women with newly diagnosed breast cancer. Cancer Nurs 2003;26:284-92.

http://www.ncbi.nlm.nih.gov/entrez/

query.fcgi? $\mathrm{cmd}=$ Retrieve $\& \mathrm{db}=$ PubMed\&list uids

$=12886119 \& \mathrm{dopt}=$ Abstract

Clar C, Waugh N, Thomas S. Routine hospital admission versus out-patient or home care in children at diagnosis of type 1 diabetes mellitus. Cochrane Database Syst Rev 2003;(3): CD004099.

http://www.ncbi.nlm.nih.gov/entrez/

query.fcgi?cmd $=$ Retrieve\&db $=$ PubMed\&list_uids

$=12918002 \& \mathrm{dopt}=$ Abstract

Clarkson JE, Worthington HV, Eden OB. Interventions for preventing oral mucositis for patients with cancer receiving treatment. Cochrane Database Syst Rev 2003;(3):CD000978.

http://www.ncbi.nlm.nih.gov/entrez/

query.fcgi?cmd = Retrieve \&db = PubMed\&list_uids

$=12917895 \& \mathrm{dopt}=$ Abstract

Cooper NJ, Sutton AJ, Abrams KR, et al. Effectiveness of neuraminidase inhibitors in treatment and prevention of influenza A and B: systematic review and meta-analyses of randomised controlled trials. BMJ 2003;326:1235-41.

http://www.ncbi.nlm.nih.gov/entrez/

query.fcgi?cmd $=$ Retrieve $\& \mathrm{db}=$ PubMed\&list_uids

$=12791735 \& \mathrm{dopt}=$ Abstract

Danne T, Aman J, Schober E, et al. A comparison of postprandial and preprandial administration of insulin aspart in children and adolescents with type 1 diabetes. Diabetes Care 2003;26:2359-64.

http://www.ncbi.nlm.nih.gov/entrez/

query.fcgi?cmd $=$ Retrieve $\& d b=$ PubMed\&list_uids

$=12882862 \& \mathrm{dopt}=$ Abstract

Davis JM, Chen N, Glick ID. A meta-analysis of the efficacy of second-generation antipsychotics. Arch Gen Psychiatry 2003; 60:553-64.

http://www.ncbi.nlm.nih.gov/entrez/

query.fcgi?cmd = Retrieve\&db = PubMed\&list_uids

$=12796218 \&$ dopt $=$ Abstract

D'Elia HF, Larsen A, Mattsson LA, et al. Influence of hormone replacement therapy on disease progression and bone mineral density in rheumatoid arthritis. J Rheumatol 2003;30:1456-63. http://www.ncbi.nlm.nih.gov/entrez/

query.fcgi?cmd $=$ Retrieve $\& \mathrm{db}=$ PubMed\&list_uids

$=12858441 \& \mathrm{dopt}=$ Abstract

De Sutter AIM, Lemiengre M, Campbell $\mathrm{H}$, et al. Antihistamines for the common cold. Cochrane Database Syst Rev 2003;(3):CD001267.

http://www.ncbi.nlm.nih.gov/entrez/

query.fcgi? $\mathrm{cmd}=$ Retrieve $\& \mathrm{db}=$ PubMed\&list_uids

$=12917904 \& \mathrm{dopt}=$ Abstract

Devereux RB, Dahlöf B, Kjeldsen SE, et al. Effects of losartan or atenolol in hypertensive patients without clinically evident vascular disease: a substudy of the LIFE randomized trial. Ann Intern Med 2003;139:169-77.

http://www.ncbi.nlm.nih.gov/entrez/

query.fcgi?cmd $=$ Retrieve $\& \mathrm{db}=$ PubMed\&list_uids

$=12899584 \& \mathrm{dopt}=$ Abstract 
Dezfulian C, Lavelle J, Nallamothu BK, et al. Rates of infection for single-lumen versus multilumen central venous catheters: a meta-analysis. Crit Care Med 2003;31:2385-90.

http://www.ncbi.nlm.nih.gov/entrez/

query.fcgi? $\mathrm{cmd}=$ Retrieve\&db $=$ PubMed\&list_uids

$=14501971 \& \mathrm{dopt}=$ Abstract

Diehl JL, Mercat A, Guérot E, et al. Helium/oxygen mixture reduces the work of breathing at the end of the weaning process in patients with severe chronic obstructive pulmonary disease. Crit Care Med 2003;31:1415-20.

http://www.ncbi.nlm.nih.gov/entrez/

query.fcgi?cmd $=$ Retrieve\&db $=$ PubMed\&list_uids

$=12771612 \& \mathrm{dopt}=$ Abstract

Drossman DA, Toner BB, Whitehead WE, et al. Cognitivebehavioral therapy versus education and desipramine versus placebo for moderate to severe functional bowel disorders. Gastroenterology 2003;125:19-31.

http://www.ncbi.nlm.nih.gov/entrez/

query.fcgi?cmd $=$ Retrieve\&db $=$ PubMed\&list_uids

$=12851867 \& \mathrm{dopt}=$ Abstract

Dugas MJ, Ladouceur R, Léger E, et al. Group cognitivebehavioral therapy for generalized anxiety disorder: treatment outcome and long-term follow-up. J Consult Clin Psychol 2003;71:821-5.

http://www.ncbi.nlm.nih.gov/entrez/

query.fcgi? $\mathrm{cmd}=$ Retrieve $\& \mathrm{db}=$ PubMed\&list_uids

$=12924687 \&$ dopt $=$ Abstract

Duncan P, Studenski S, Richards L, et al. Randomized clinical trial of therapeutic exercise in subacute stroke. Stroke 2003;34:2173-80.

http://www.ncbi.nlm.nih.gov/entrez/

query.fcgi? $\mathrm{cmd}=$ Retrieve $\& \mathrm{db}=$ PubMed\&list_uids

$=12920254 \& \mathrm{dopt}=$ Abstract

Edmonds ML, Camargo CA Jr, Pollack CV Jr, et al. Early use of inhaled corticosteroids in the emergency department treatment of acute asthma. Cochrane Database Syst Rev 2003;(3):CD002308. http://www.ncbi.nlm.nih.gov/entrez/

query.fcgi?cmd $=$ Retrieve $\& \mathrm{db}=$ PubMed\&list_uids

$=12917930 \& \mathrm{dopt}=$ Abstract

Edwards A, Unigwe S, Elwyn G, et al. Effects of communicating individual risks in screening programmes: Cochrane systematic review. BMJ 2003;327:703-9.

http://www.ncbi.nlm.nih.gov/entrez/

query.fcgi?cmd $=$ Retrieve $\& \mathrm{db}=$ PubMed\&list_uids

$=14512475 \& \mathrm{dopt}=$ Abstract

Ellertson C, Webb A, Blanchard K, et al. Modifying the Yuzpe regimen of emergency contraception: a multicenter randomized controlled trial. Obstet Gynecol 2003;101:1160-7.

http://www.ncbi.nlm.nih.gov/entrez/

query.fcgi?cmd $=$ Retrieve\&db $=$ PubMed\&list_uids

$=12798518 \& \mathrm{dopt}=$ Abstract

Ellström MA, Aström M, Möller A, et al. A randomized trial comparing changes in psychological well-being and sexuality after laparoscopic and abdominal hysterectomy. Acta Obstet Gynecol Scand 2003;82:871-5.

http://www.ncbi.nlm.nih.gov/entrez/

query.fcgi? $\mathrm{cmd}=$ Retrieve $\& \mathrm{db}=$ PubMed\&list_uids

$=12911451 \& \mathrm{dopt}=$ Abstract

Faggiano F, Vigna-Taglianti F, Versino E, et al. Methadone maintenance at different dosages for opioid dependence. Cochrane Database Syst Rev 2003;(3):CD002208. http://www.ncbi.nlm.nih.gov/entrez/

query.fcgi?cmd $=$ Retrieve $\& d b=$ PubMed\&list_uids

$=12917925 \&$ dopt $=$ Abstract

Feldman H, Gauthier S, Hecker J, et al. Efficacy of donepezil on maintenance of activities of daily living in patients with moderate to severe Alzheimer's disease and the effect on caregiver burden. J Am Geriatr Soc 2003;51:737-44.

http://www.ncbi.nlm.nih.gov/entrez/

query.fcgi?cmd $=$ Retrieve\&db $=$ PubMed\&list_uids

$=12757558 \& \mathrm{dopt}=$ Abstract

Ferrer M, Esquinas A, Arancibia F, et al. Noninvasive ventilation during persistent weaning failure: a randomized controlled trial. Am J Respir Crit Care Med 2003;168:70-6.

http://www.ncbi.nlm.nih.gov/entrez/

query.fcgi?cmd $=$ Retrieve $\& \mathrm{db}=$ PubMed\&list_uids

$=12689847 \& \mathrm{dopt}=$ Abstract

Ferri M, Davoli M, Perucci CA. Heroin maintenance for chronic heroin dependents. Cochrane Database Syst Rev 2003;(3):CD003410.

http://www.ncbi.nlm.nih.gov/entrez/

query.fcgi?cmd $=$ Retrieve $\& \mathrm{db}=$ PubMed\&list_uids

$=12917967 \&$ dopt $=$ Abstract

Frame K, Kelly L, Bayley E. Increasing perceptions of selfworth in preadolescents diagnosed with ADHD. J Nurs Scholarsh 2003;35:225-9.

http://www.ncbi.nlm.nih.gov/entrez/

query.fcgi?cmd $=$ Retrieve $\& \mathrm{db}=$ PubMed\&list_uids

$=14562489 \& \mathrm{dopt}=$ Abstract

Frey SE, Newman FK, Yan L, et al. Response to smallpox vaccine in persons immunized in the distant past. JAMA 2003;289:3295-9.

http://www.ncbi.nlm.nih.gov/entrez/

query.fcgi? $\mathrm{cmd}=$ Retrieve $\& \mathrm{db}=$ PubMed\&list_uids

$=12824212 \& \mathrm{dopt}=$ Abstract

Fransen M, McConnell S, Bell M. Exercise for osteoarthritis of the hip or knee. Cochrane Database Syst Rev 2003;(3): CD004286.

http://www.ncbi.nlm.nih.gov/entrez/

query.fcgi? $\mathrm{cmd}=$ Retrieve $\& \mathrm{db}=$ PubMed\&list_uids

$=12918008 \& \mathrm{dopt}=$ Abstract

French J, Bilton D, Campbell F. Nurse specialist care for bronchiectasis. Cochrane Database Syst Rev 2003;(3):CD004319. http://www.ncbi.nlm.nih.gov/entrez/

query.fcgi?cmd $=$ Retrieve $\& \mathrm{db}=$ PubMed\&list_uids

$=12918011 \& \mathrm{dopt}=$ Abstract

Fudala PJ, Bridge TP, Herbert S, et al. Office-based treatment of opiate addiction with a sublingual-tablet formulation of buprenorphine and naloxone. N Engl J Med 2003;349:949-58. http://www.ncbi.nlm.nih.gov/entrez/

query.fcgi? $\mathrm{cmd}=$ Retrieve\&db $=$ PubMed\&list_uids

$=12954743 \& \mathrm{dopt}=$ Abstract

Furukawa T, McGuire H, Barbui C. Low dosage tricyclic antidepressants for depression. Cochrane Database Syst Rev 2003; (3):CD003197.

http://www.ncbi.nlm.nih.gov/entrez/

query.fcgi?cmd $=$ Retrieve $\& \mathrm{db}=$ PubMed\&list_uids

$=12917952 \& \mathrm{dopt}=$ Abstract

George A, Rubin G. A systematic review and meta-analysis of treatments for impetigo. Br J Gen Pract 2003;53:480-7. 
4 of 14

http://www.ncbi.nlm.nih.gov/entrez/

query.fcgi?cmd $=$ Retrieve $\& \mathrm{db}=$ PubMed\&list_uids

$=12939895 \& \mathrm{dopt}=$ Abstract

Glasgow NJ, Ponsonby AL, Yates R, et al. Proactive asthma care in childhood: general practice based randomised controlled trial. BMJ 2003;327:659-65.

http://www.ncbi.nlm.nih.gov/entrez/

query.fcgi?cmd $=$ Retrieve $\& \mathrm{db}=$ PubMed\&list_uids

$=14500440 \& \mathrm{dopt}=$ Abstract

Glazener CMA, Evans JHC, Peto RE. Tricyclic and related drugs for nocturnal enuresis in children. Cochrane Database Syst Rev 2003;(3):CD002117.

http://www.ncbi.nlm.nih.gov/entrez/

query.fcgi?cmd $=$ Retrieve\&db $=$ PubMed\&list_uids

$=12917922 \& \mathrm{dopt}=$ Abstract

Goldbeck L, Schmid K. Effectiveness of autogenic relaxation training on children and adolescents with behavioral and emotional problems. J Am Acad Child Adolesc Psychiatry 2003;42:1046-54.

http://www.ncbi.nlm.nih.gov/entrez/

query.fcgi?cmd $=$ Retrieve \&db $=$ PubMed\&list_uids

$=12960704 \& \mathrm{dopt}=$ Abstract

Guevara JP, Wolf FM, Grum CM, et al. Effects of educational interventions for self management of asthma in children and adolescents: systematic review and meta-analysis. BMJ 2003;326:1308-13.

http://www.ncbi.nlm.nih.gov/entrez/

query.fcgi?cmd $=$ Retrieve $\& \mathrm{db}=$ PubMed\&list_uids

$=12805167 \& \mathrm{dopt}=$ Abstract

Hamann A, Matthaei S, Rosak C, et al. A randomized clinical trial comparing breakfast, dinner, or bedtime administration of insulin glargine in patients with type 1 diabetes. Diabetes Care 2003;26:1738-44.

http://www.ncbi.nlm.nih.gov/entrez/

query.fcgi?cmd $=$ Retrieve\&db $=$ PubMed\&list_uids

$=12766103 \& \mathrm{dopt}=$ Abstract

Hawkins G, McMahon AD, Twaddle S, et al. Stepping down inhaled corticosteroids in asthma: randomised controlled trial. BMJ 2003;326:1115-21.

http://www.ncbi.nlm.nih.gov/entrez/

query.fcgi?cmd $=$ Retrieve \&db = PubMed\&list_uids

$=12763981 \& \mathrm{dopt}=$ Abstract

Hedayati H, Parsons J, Crowther CA. Rectal analgesia for pain from perineal trauma following childbirth. Cochrane Database Syst Rev 2003;(3):CD003931.

http://www.ncbi.nlm.nih.gov/entrez/

query.fcgi?cmd $=$ Retrieve $\& \mathrm{db}=$ PubMed\&list_uids

$=12917995 \& \mathrm{dopt}=$ Abstract

Hind D, Calvert N, McWilliams R, et al. Ultrasonic locating devices for central venous cannulation: meta-analysis. BMJ 2003;327:361-7.

http://www.ncbi.nlm.nih.gov/entrez/

query.fcgi?cmd $=$ Retrieve $\& \mathrm{db}=$ PubMed\&list_uids

$=12919984 \& \mathrm{dopt}=$ Abstract

Hinman RS, Crossley KM, McConnell J, et al. Efficacy of knee tape in the management of osteoarthritis of the knee: blinded randomised controlled trial. BMJ 2003;327:135-40.

http://www.ncbi.nlm.nih.gov/entrez/

query.fcgi?cmd $=$ Retrieve \&db = PubMed\&list_uids

$=12869456 \& \mathrm{dopt}=$ Abstract
Hodnett ED, Fredericks S. Support during pregnancy for women at increased risk of low birthweight babies. Cochrane Database Syst Rev 2003;(3):CD000198.

http://www.ncbi.nlm.nih.gov/entrez/

query.fcgi?cmd $=$ Retrieve $\& \mathrm{db}=$ PubMed\&list_uids

$=12917888 \& \mathrm{dopt}=$ Abstract

Hodnett ED, Gates S, Hofmeyr GJ, et al. Continuous support for women during childbirth. Cochrane Database Syst Rev 2003; (3):CD003766.

http://www.ncbi.nlm.nih.gov/entrez/

query.fcgi?cmd = Retrieve \&db = PubMed\&list_uids

$=12917986 \& \mathrm{dopt}=$ Abstract

Hoffman DR, Birch EE, Castañeda YS, et al. Visual function in breast-fed term infants weaned to formula with or without long-chain polyunsaturates at 4 to 6 months: a randomized clinical trial. J Pediatr 2003;142:669-77.

http://www.ncbi.nlm.nih.gov/entrez/

query.fcgi?cmd $=$ Retrieve $\& \mathrm{db}=$ PubMed\&list_uids

$=12838196 \& \mathrm{dopt}=$ Abstract

Holloway RM, Wilkinson C, Peters TJ, et al. Clusterrandomised trial of risk communication to enhance informed uptake of cervical screening. Br J Gen Pract 2003;53:620-5. http://www.ncbi.nlm.nih.gov/entrez/

query.fcgi?cmd $=$ Retrieve $\& \mathrm{db}=$ PubMed\&list_uids $=14601338 \& \mathrm{dopt}=$ Abstract

Hooper L, Bartlett C, Davey Smith G, et al. Reduced dietary salt for prevention of cardiovascular disease. Cochrane Database Syst Rev 2003;(3):CD003656.

http://www.ncbi.nlm.nih.gov/entrez/

query.fcgi?cmd $=$ Retrieve $\& d b=$ PubMed\&list_uids

$=12917977 \& \mathrm{dopt}=$ Abstract

Hosoda K, Wang MF, Liao ML, et al. Antihyperglycemic effect of oolong tea in type 2 diabetes. Diabetes Care 2003;26:1714-8. http://www.ncbi.nlm.nih.gov/entrez/

query.fcgi?cmd = Retrieve\&db = PubMed\&list_uids

$=12766099 \& \mathrm{dopt}=$ Abstract

Jakicic JM, Marcus BH, Gallagher KI, et al. Effect of exercise duration and intensity on weight loss in overweight, sedentary women: a randomized trial. JAMA 2003;290:1323-30.

http://www.ncbi.nlm.nih.gov/entrez/

query.fcgi?cmd $=$ Retrieve $\& \mathrm{db}=$ PubMed\&list_uids

$=12966123 \&$ dopt $=$ Abstract

Jenkins DJ, Kendall CW, Marchie A, et al. Effects of a dietary portfolio of cholesterol-lowering foods vs lovastatin on serum lipids and C-reactive protein. JAMA 2003;290:502-10.

http://www.ncbi.nlm.nih.gov/entrez/

query.fcgi cmd $=$ Retrieve $\& d b=$ PubMed\&list_uids

$=12876093 \& \mathrm{dopt}=$ Abstract

Jibaja-Weiss ML, Volk RJ, Kingery P, et al. Tailored messages for breast and cervical cancer screening of low-income and minority women using medical records data. Patient Educ Couns 2003;50:123-32.

http://www.ncbi.nlm.nih.gov/entrez/

query.fcgi?cmd $=$ Retrieve\&db $=$ PubMed\&list_uids

$=12781927 \& \mathrm{dopt}=$ Abstract

Klerk CP, Smorenburg SM, Büller HR. Thrombosis prophylaxis in patient populations with a central venous catheter: a systematic review. Arch Intern Med 2003;163:1913-21.

http://www.ncbi.nlm.nih.gov/entrez/

query.fcgi?cmd $=$ Retrieve $\& \mathrm{db}=$ PubMed\&list_uids

$=12963564 \& \mathrm{dopt}=$ Abstract 
Labarere J, Bellin V, Fourny $\mathrm{M}$, et al. Assessment of a structured in-hospital educational intervention addressing breastfeeding: a prospective randomised open trial. BJOG 2003; 110:847-52.

http://www.ncbi.nlm.nih.gov/entrez/

query.fcgi?cmd $=$ Retrieve\&db $=$ PubMed\&list_uids

$=14511968 \& \mathrm{dopt}=$ Abstract

Latham NK, Anderson CS, Reid IR. Effects of vitamin D supplementation on strength, physical performance, and falls in older persons: a systematic review. J Am Geriatr Soc 2003;51:1219-26.

http://www.ncbi.nlm.nih.gov/entrez/

query.fcgi?cmd $=$ Retrieve $\& \mathrm{db}=$ PubMed\&list_uids

$=12919233 \& \mathrm{dopt}=$ Abstract

Law J, Garrett Z, Nye C. Speech and language therapy interventions for children with primary speech and language delay or disorder. Cochrane Database Syst Rev 2003;(3):CD004110.

http://www.ncbi.nlm.nih.gov/entrez/

query.fcgi?cmd $=$ Retrieve\&db $=$ PubMed\&list_uids

$=12918003 \& \mathrm{dopt}=$ Abstract

Law MR, Wald NJ, Rudnicka AR. Quantifying effect of statins on low density lipoprotein cholesterol, ischaemic heart disease, and stroke: systematic review and meta-analysis. BMJ 2003;326:1423-9.

http://www.ncbi.nlm.nih.gov/entrez/

query.fcgi? cmd $=$ Retrieve\&db $=$ PubMed\&list_uids

$=12829554 \& \mathrm{dopt}=$ Abstract

Lee AY, Levine MN, Baker RI, et al. Low-molecular-weight heparin versus a coumarin for the prevention of recurrent venous thromboembolism in patients with cancer. $N$ Engl $J$ Med 2003;349:146-53.

http://www.ncbi.nlm.nih.gov/entrez/

query.fcgi?cmd $=$ Retrieve\&db $=$ PubMed\&list_uids

$=12853587 \& \mathrm{dopt}=$ Abstract

León C, Alvarez-Lerma F, Ruiz-Santana S, et al. Antiseptic chamber-containing hub reduces central venous catheterrelated infection: a prospective, randomized study. Crit Care Med 2003;31:1318-24.

http://www.ncbi.nlm.nih.gov/entrez/

query.fcgi?cmd $=$ Retrieve\&db $=$ PubMed\&list_uids

$=12771597 \&$ dopt $=$ Abstract

Leucht S, Barnes TR, Kissling W, et al. Relapse prevention in schizophrenia with new-generation antipsychotics: a systematic review and exploratory meta-analysis of randomized, controlled trials. Am J Psychiatry 2003;160:1209-22.

http://www.ncbi.nlm.nih.gov/entrez/

query.fcgi? $\mathrm{cmd}=$ Retrieve $\& \mathrm{db}=$ PubMed\&list_uids

$=12832232 \& \mathrm{dopt}=$ Abstract

Levine $\mathrm{CB}$, Fahrbach KR, Siderowf AD, et al. Diagnosis and treatment of Parkinson's Disease: a systematic review of the literature. Evidence Report/Technology Assessment Number 57. AHRQ Publication No. 03-E040. Rockville, MD: Agency for Healthcare Research and Quality, June 2003.

http://www.ahrq.gov/clinic/parkinv.htm

Lisse JR, Perlman M, Johansson G, et al. Gastrointestinal tolerability and effectiveness of rofecoxib versus naproxen in the treatment of osteoarthritis: a randomized, controlled trial. Ann Intern Med 2003;139:539-46.

http://www.ncbi.nlm.nih.gov/entrez/

query.fcgi?cmd $=$ Retrieve\&db $=$ PubMed\&list_uids

$=14530224 \& \mathrm{dopt}=$ Abstract
Littrell KH, Hilligoss NM, Kirshner CD, et al. The effects of an educational intervention on antipsychotic-induced weight gain. J Nurs Scholarsh 2003;35:237-41

http://www.ncbi.nlm.nih.gov/entrez/

query.fcgi?cmd $=$ Retrieve $\& d b=$ PubMed\&list_uids

$=14562491 \& \mathrm{dopt}=$ Abstract

Loeb MB, Becker M, Eady A, et al. Interventions to prevent aspiration pneumonia in older adults: a systematic review. $J$ Am Geriatr Soc 2003;51:1018-22.

http://www.ncbi.nlm.nih.gov/entrez/

query.fcgi?cmd $=$ Retrieve $\& \mathrm{db}=$ PubMed\&list_uids

$=12834525 \& \mathrm{dopt}=$ Abstract

Lorente L, Lecuona M, Málaga J, et al. Bacterial filters in respiratory circuits: an unnecessary cost? Crit Care Med 2003;31:2126-30.

http://www.ncbi.nlm.nih.gov/entrez/

query.fcgi?cmd = Retrieve \&db = PubMed\&list_uids

$=12973169 \& \mathrm{dopt}=$ Abstract

Lyketsos CG, DelCampo L, Steinberg M, et al. Treating depression in Alzheimer disease: efficacy and safety of sertraline therapy, and the benefits of depression reduction: the DIADS. Arch Gen Psychiatry 2003;60:737-46.

http://www.ncbi.nlm.nih.gov/entrez/

query.fcgi?cmd $=$ Retrieve $\& \mathrm{db}=$ PubMed\&list_uids

$=12860778 \& \mathrm{dopt}=$ Abstract

Lysy J, Sistiery-Ittah M, Israelit Y, et al. Topical capsaicin - a novel and effective treatment for idiopathic intractable pruritus ani: a randomised, placebo controlled, crossover study. Gut 2003;52:1323-6.

http://www.ncbi.nlm.nih.gov/entrez/

query.fcgi? $\mathrm{cmd}=$ Retrieve $\& \mathrm{db}=$ PubMed\&list_uids

$=12912865 \& \mathrm{dopt}=$ Abstract

Malouf R, Areosa Sastre A. Vitamin B12 for cognition. Cochrane Database Syst Rev 2003;(3):CD004326.

http://www.ncbi.nlm.nih.gov/entrez/

query.fcgi?cmd $=$ Retrieve $\& \mathrm{db}=$ PubMed\&list_uids

$=12918012 \& \mathrm{dopt}=$ Abstract

Manderson JG, Patterson CC, Hadden DR, et al. Preprandial versus postprandial blood glucose monitoring in type 1 diabetic pregnancy: a randomized controlled clinical trial. Am J Obstet Gynecol 2003;189:507-12.

http://www.ncbi.nlm.nih.gov/entrez/

query.fcgi?cmd $=$ Retrieve $\& \mathrm{db}=$ PubMed\&list_uids

$=14520226 \& \mathrm{dopt}=$ Abstract

Maron DJ, Lu GP, Cai NS, et al. Cholesterol-lowering effect of a theaflavin-enriched green tea extract: a randomized controlled trial. Arch Intern Med 2003;163:1448-53.

http://www.ncbi.nlm.nih.gov/entrez/

query.fcgi?cmd $=$ Retrieve $\& \mathrm{db}=$ PubMed\&list_uids

$=12824094 \& \mathrm{dopt}=$ Abstract

Marriott LD, Foote KD, Bishop JA, et al. Weaning preterm infants: a randomised controlled trial. Arch Dis Child Fetal Neonatal Ed 2003;88:F302-7.

http://www.ncbi.nlm.nih.gov/entrez/

query.fcgi?cmd $=$ Retrieve $\& \mathrm{db}=$ PubMed\&list_uids

$=12819162 \& \mathrm{dopt}=$ Abstract

Marston WA, Hanft J, Norwood P, et al. The efficacy and safety of Dermagraft in improving the healing of chronic diabetic foot ulcers: results of a prospective randomized trial. Diabetes Care 2003;26:1701-5. 
6 of 14

http://www.ncbi.nlm.nih.gov/entrez/

query.fcgi?cmd $=$ Retrieve $\& \mathrm{db}=$ PubMed\&list_uids

$=12766097 \& \mathrm{dopt}=$ Abstract

Matheson NJ, Symmonds-Abrahams M, Sheikh A, et al. Neuraminidase inhibitors for preventing and treating influenza in children. Cochrane Database Syst Rev 2003;(3):CD002744.

http://www.ncbi.nlm.nih.gov/entrez/

query.fcgi? $\mathrm{cmd}=$ Retrieve $\& \mathrm{db}=$ PubMed\&list_uids $=12917931 \& \mathrm{dopt}=$ Abstract

McCrory DC, Gray RN. Oral sumatriptan for acute migraine. Cochrane Database Syst Rev 2003;(3):CD002915.

http://www.ncbi.nlm.nih.gov/entrez/

query.fcgi?cmd $=$ Retrieve $\& \mathrm{db}=$ PubMed\&list_uids

$=12917936 \& \mathrm{dopt}=$ Abstract

Miranda J, Chung JY, Green BL, et al. Treating depression in predominantly low-income young minority women: a randomized controlled trial. JAMA 2003;290:57-65.

http://www.ncbi.nlm.nih.gov/entrez/

query.fcgi? $\mathrm{cmd}=$ Retrieve \&db $=$ PubMed\&list_uids

$=12837712 \& \mathrm{dopt}=$ Abstract

Moayyedi P, Soo S, Deeks J, et al. Systematic review: antacids, H2-receptor antagonists, prokinetics, bismuth and sucralfate therapy for non-ulcer dyspepsia. Aliment Pharmacol Ther 2003; 17:1215-27.

http://www.ncbi.nlm.nih.gov/entrez/

query.fcgi?cmd $=$ Retrieve $\& \mathrm{db}=$ PubMed\&list_uids

$=12755835 \& \mathrm{dopt}=$ Abstract

Mohler ER 3rd, Hiatt WR, Creager MA. Cholesterol reduction with atorvastatin improves walking distance in patients with peripheral arterial disease. Circulation 2003;108:1481-6.

http://www.ncbi.nlm.nih.gov/entrez/

query.fcgi?cmd $=$ Retrieve\&db $=$ PubMed\&list_uids

$=12952839 \& \mathrm{dopt}=$ Abstract

Mohr DC, Hart SL, Goldberg A. Effects of treatment for depression on fatigue in multiple sclerosis. Psychosom Med 2003;65:542-7.

http://www.ncbi.nlm.nih.gov/entrez/

query.fcgi?cmd $=$ Retrieve $\& \mathrm{db}=$ PubMed\&list_uids

$=12883103 \& \mathrm{dopt}=$ Abstract

Molyneux A, Lewis S, Leivers U, et al. Clinical trial comparing nicotine replacement therapy (NRT) plus brief counselling, brief counselling alone, and minimal intervention on smoking cessation in hospital inpatients. Thorax 2003;58:484-8.

http://www.ncbi.nlm.nih.gov/entrez/

query.fcgi?cmd $=$ Retrieve $\& \mathrm{db}=$ PubMed\&list_uids

$=12775857 \& \mathrm{dopt}=$ Abstract

Morris CD, Carson S. Routine vitamin supplementation to prevent cardiovascular disease: a summary of the evidence for the U.S. Preventive Services Task Force. Ann Intern Med 2003; 139:56-70.

http://www.ncbi.nlm.nih.gov/entrez/

query.fcgi?cmd $=$ Retrieve\&db $=$ PubMed\&list_uids

$=12834320 \& \mathrm{dopt}=$ Abstract

Murphy KR, Fitzpatrick S, Cruz-Rivera M, et al. Effects of budesonide inhalation suspension compared with cromolyn sodium nebulizer solution on health status and caregiver quality of life in childhood asthma. Pediatrics 2003;112:e212-9. http://www.ncbi.nlm.nih.gov/entrez/ query.fcgi?cmd $=$ Retrieve \&db $=$ PubMed\&list_uids $=12949315 \&$ dopt $=$ Abstract
Nordhus IH, Pallesen S. Psychological treatment of late-life anxiety: an empirical review. $J$ Consult Clin Psychol 2003;71:643-51.

http://www.ncbi.nlm.nih.gov/entrez/

query.fcgi? $\mathrm{cmd}=$ Retrieve $\& \mathrm{db}=$ PubMed\&list_uids

$=12924668 \& \mathrm{dopt}=$ Abstract

Nosé M, Barbui C, Gray R, et al. Clinical interventions for treatment non-adherence in psychosis: meta-analysis. $\mathrm{Br} \mathrm{J}$ Psychiatry 2003;183:197-206.

http://www.ncbi.nlm.nih.gov/entrez/

query.fcgi?cmd = Retrieve\&db = PubMed\&list_uids

$=12948991 \&$ dopt $=$ Abstract

O'Brien KL, Moulton LH, Reid R, et al. Efficacy and safety of seven-valent conjugate pneumococcal vaccine in American Indian children: group randomised trial. Lancet 2003;362:355-61.

http://www.ncbi.nlm.nih.gov/entrez/

query.fcgi?cmd $=$ Retrieve $\& \mathrm{db}=$ PubMed\&list_uids

$=12907008 \& \mathrm{dopt}=$ Abstract

O'Malley SS, Rounsaville BJ, Farren C, et al. Initial and maintenance naltrexone treatment for alcohol dependence using primary care vs specialty care: a nested sequence of 3 randomized trials. Arch Intern Med 2003;163:1695-704.

http://www.ncbi.nlm.nih.gov/entrez/

query.fcgi?cmd $=$ Retrieve $\& \mathrm{db}=$ PubMed\&list_uids

$=12885685 \& \mathrm{dopt}=$ Abstract

Paradise JL, Dollaghan CA, Campbell TF, et al. Otitis media and tympanostomy tube insertion during the first three years of life: developmental outcomes at the age of four years. Pediatrics 2003;112:265-77.

http://www.ncbi.nlm.nih.gov/entrez/

query.fcgi?cmd $=$ Retrieve $\& \mathrm{db}=$ PubMed\&list_uids

$=12897272 \& \mathrm{dopt}=$ Abstract

Pedersen T, Dyrlund Pedersen B, Moller AM. Pulse oximetry for perioperative monitoring. Cochrane Database Syst Rev 2003;(3):CD002013.

http://www.ncbi.nlm.nih.gov/entrez/

query.fcgi?cmd $=$ Retrieve $\& \mathrm{db}=$ PubMed\&list_uids

$=12917918 \& \mathrm{dopt}=$ Abstract

Plank J, Haas W, Rakovac I, et al. Evaluation of the impact of chiropodist care in the secondary prevention of foot ulcerations in diabetic subjects. Diabetes Care 2003;26:1691-5.

http://www.ncbi.nlm.nih.gov/entrez/

query.fcgi?cmd $=$ Retrieve $\& \mathrm{db}=$ PubMed\&list_uids

$=12766095 \& \mathrm{dopt}=$ Abstract

Poole-Wilson PA, Swedberg K, Cleland JG, et al. Comparison of carvedilol and metoprolol on clinical outcomes in patients with chronic heart failure in the Carvedilol Or Metoprolol European Trial (COMET): randomised controlled trial. Lancet 2003;362:7-13.

http://www.ncbi.nlm.nih.gov/entrez/

query.fcgi?cmd = Retrieve\&db = PubMed\&list_uids

$=12853193 \& \mathrm{dopt}=$ Abstract

Priest SR, Henderson J, Evans SF, et al. Stress debriefing after childbirth: a randomised controlled trial. Med J Aust 2003; 178:542-5.

http://www.ncbi.nlm.nih.gov/entrez/

query.fcgi? $\mathrm{cmd}=$ Retrieve $\& \mathrm{db}=$ PubMed\&list_uids

$=12765500 \& \mathrm{dopt}=$ Abstract

Pshetizky Y, Naimer S, Shvartzman P. Acute otitis media-a brief explanation to parents and antibiotic use. Fam Pract 2003;20:417-9. 
http://www.ncbi.nlm.nih.gov/entrez/

query.fcgi?cmd $=$ Retrieve\&db $=$ PubMed\&list_uids

$=12876113 \& \mathrm{dopt}=$ Abstract

Rahmathullah L, Tielsch JM, Thulasiraj RD, et al. Impact of supplementing newborn infants with vitamin A on early infant mortality: community based randomised trial in southern India. BMJ 2003;327:254-9.

http://www.ncbi.nlm.nih.gov/entrez/

query.fcgi?cmd $=$ Retrieve $\& \mathrm{db}=$ PubMed\&list_uids

$=12896935 \& \mathrm{dopt}=$ Abstract

Rehse B, Pukrop R. Effects of psychosocial interventions on quality of life in adult cancer patients: meta analysis of 37 published controlled outcome studies. Patient Educ Couns 2003;50:179-86.

http://www.ncbi.nlm.nih.gov/entrez/

query.fcgi? $\mathrm{cmd}=$ Retrieve $\& \mathrm{db}=$ PubMed\&list_uids

$=12781933 \& \mathrm{dopt}=$ Abstract

Rejeski WJ, Brawley LR, Ambrosius WT, et al. Older adults with chronic disease: benefits of group-mediated counseling in the promotion of physically active lifestyles. Health Psychol 2003;22:414-23.

http://www.ncbi.nlm.nih.gov/entrez/

query.fcgi?cmd $=$ Retrieve \&db $=$ PubMed\&list_uids

$=12940398 \& \mathrm{dopt}=$ Abstract

Richter B, Neises G. 'Human' insulin versus animal insulin in people with diabetes mellitus. Cochrane Database Syst Rev 2003;(3):CD003816.

http://www.ncbi.nlm.nih.gov/entrez/

query.fcgi? $\mathrm{cmd}=$ Retrieve $\& \mathrm{db}=$ PubMed\&list_uids

$=12917989 \& \mathrm{dopt}=$ Abstract

Richy F, Bruyere O, Ethgen O, et al. Structural and symptomatic efficacy of glucosamine and chondroitin in knee osteoarthritis: a comprehensive meta-analysis. Arch Intern Med 2003;163:1514-22.

http://www.ncbi.nlm.nih.gov/entrez/

query.fcgi?cmd $=$ Retrieve $\& \mathrm{db}=$ PubMed\&list_uids

$=12860572 \& \mathrm{dopt}=$ Abstract

Roseby R, Waters E, Polnay A, et al. Family and carer smoking control programmes for reducing children's exposure to environmental tobacco smoke. Cochrane Database Syst Rev 2003;(3):CD001746.

http://www.ncbi.nlm.nih.gov/entrez/

query.fcgi?cmd $=$ Retrieve \&db $=$ PubMed\&list_uids

$=12917911 \& \mathrm{dopt}=$ Abstract

Roux C, Kolta S, Desfougères JL, et al. Long-term safety of fluticasone propionate and nedocromil sodium on bone in children with asthma. Pediatrics 2003;111:e706-13.

http://www.ncbi.nlm.nih.gov/entrez/

query.fcgi?cmd $=$ Retrieve $\& \mathrm{db}=$ PubMed\&list_uids

$=12777589 \& \mathrm{dopt}=$ Abstract

Royle P, Waugh N, McAuley L, et al. Inhaled insulin in diabtetes mellitus. Cochrane Database Syst Rev 2003; (3):CD003890.

http://www.ncbi.nlm.nih.gov/entrez/

query.fcgi?cmd $=$ Retrieve \&db $=$ PubMed\&list_uids

$=12917994 \& \mathrm{dopt}=$ Abstract

Sarrell EM, Cohen HA, Kahan E. Naturopathic treatment for ear pain in children. Pediatrics 2003;111:e574-9.

http://www.ncbi.nlm.nih.gov/entrez/

query.fcgi?cmd $=$ Retrieve\&db $=$ PubMed\&list_uids

$=12728112 \& \mathrm{dopt}=$ Abstract
Schneider LS, Nelson JC, Clary CM, et al. An 8-week multicenter, parallel-group, double-blind, placebo-controlled study of sertraline in elderly outpatients with major depression. Am J Psychiatry 2003;160:1277-85.

http://www.ncbi.nlm.nih.gov/entrez/

query.fcgi?cmd $=$ Retrieve $\& \mathrm{db}=$ PubMed\&list_uids

$=12832242 \& \mathrm{dopt}=$ Abstract

Scott JT, Harmsen M, Prictor MJ, et al. Interventions for improving communication with children and adolescents about their cancer. Cochrane Database Syst Rev 2003;(3):CD002969. http://www.ncbi.nlm.nih.gov/entrez/

query.fcgi?cmd $=$ Retrieve $\& \mathrm{db}=$ PubMed\&list_uids

$=12917938 \& \mathrm{dopt}=$ Abstract

Segal JB, Bolger DT, Jenckes MW, et al. Outpatient therapy with low molecular weight heparin for the treatment of venous thromboembolism: a review of efficacy, safety, and costs. Am J Med 2003;115:298-308.

http://www.ncbi.nlm.nih.gov/entrez/

query.fcgi?cmd $=$ Retrieve $\& d b=$ PubMed\&list_uids

$=12967695 \& \mathrm{dopt}=$ Abstract

Sellors J, Kaczorowski J, Sellors C, et al. A randomized controlled trial of a pharmacist consultation program for family physicians and their elderly patients. CMAJ 2003; 169:17-22.

http://www.ncbi.nlm.nih.gov/entrez/

query.fcgi? $\mathrm{cmd}=$ Retrieve $\& \mathrm{db}=$ PubMed\&list_uids

$=12847034 \& \mathrm{dopt}=$ Abstract

Shabnam GN, Chung TH, Deane KHO, et al. Therapies for depression in Parkinson's Disease. Cochrane Database Syst Rev 2003;(3):CD003465.

http://www.ncbi.nlm.nih.gov/entrez/

query.fcgi?cmd $=$ Retrieve $\& \mathrm{db}=$ PubMed\&list_uid-

$\mathrm{s}=12917968 \& \mathrm{dopt}=$ Abstract

Shah L, Wilson AJ, Gibson PG, et al. Long acting betaagonists versus theophylline for maintenance treatment of asthma. Cochrane Database Syst Rev 2003;(3):CD001281.

http://www.ncbi.nlm.nih.gov/entrez/

query.fcgi?cmd $=$ Retrieve $\& \mathrm{db}=$ PubMed\&list_uids

$=12917905 \& \mathrm{dopt}=$ Abstract

Shlay JC, Mayhugh B, Foster M, et al. Initiating contraception in sexually transmitted disease clinic setting: a randomized trial. Am J Obstet Gynecol 2003;189:473-81.

http://www.ncbi.nlm.nih.gov/entrez/

query.fcgi?cmd = Retrieve\&db = PubMed\&list uids

$=14520221 \& \mathrm{dopt}=$ Abstract

Simon JA, Carmody TP, Hudes ES, et al. Intensive smoking cessation counseling versus minimal counseling among hospitalized smokers treated with transdermal nicotine replacement: a randomized trial. Am J Med 2003;114:555-62. http://www.ncbi.nlm.nih.gov/entrez/

query.fcgi?cmd $=$ Retrieve $\& \mathrm{db}=$ PubMed\&list uids

$=12753879 \& \mathrm{dopt}=$ Abstract

Snowden M, Sato K, Roy-Byrne P. Assessment and treatment of nursing home residents with depression or behavioral symptoms associated with dementia: a review of the literature. J Am Geriatr Soc 2003;51:1305-17.

http://www.ncbi.nlm.nih.gov/entrez/

query.fcgi? $\mathrm{cmd}=$ Retrieve $\& \mathrm{db}=$ PubMed\&list_uids

$=12919245 \& \mathrm{dopt}=$ Abstract

Steed L, Cooke D, Newman S. A systematic review of psychosocial outcomes following education, self-management 
8 of 14

and psychological interventions in diabetes mellitus. Patient Educ Couns 2003;51:5-15.

http://www.ncbi.nlm.nih.gov/entrez/

query.fcgi? $\mathrm{cmd}=$ Retrieve $\& \mathrm{db}=$ PubMed\&list_uids

$=12915275 \& \mathrm{dopt}=$ Abstract

Stein BD, Jaycox LH, Kataoka SH, et al. A mental health intervention for schoolchildren exposed to violence: a randomized controlled trial. JAMA 2003;290:603-11.

http://www.ncbi.nlm.nih.gov/entrez/

query.fcgi?cmd $=$ Retrieve\&db $=$ PubMed\&list_uids

$=12902363 \& \mathrm{dopt}=$ Abstract

Subhedar NV, Shaw NJ. Dopamine versus dobutamine for hypotensive preterm infants. Cochrane Database Syst Rev 2003;(3):CD001242.

http://www.ncbi.nlm.nih.gov/entrez/

query.fcgi?cmd $=$ Retrieve $\& \mathrm{db}=$ PubMed\&list_uids

$=12917901 \& \mathrm{dopt}=$ Abstract

Summerbell CD, Ashton V, Campbell KJ, et al. Interventions for treating obesity in children. Cochrane Database Syst Rev 2003;(3):CD001872.

http://www.ncbi.nlm.nih.gov/entrez/

query.fcgi? $\mathrm{cmd}=$ Retrieve $\& \mathrm{db}=$ PubMed\&list_uids

$=12917914 \& \mathrm{dopt}=$ Abstract

Tam WH, Lee DT, Chiu HF, et al. A randomised controlled trial of educational counselling on the management of women who have suffered suboptimal outcomes in pregnancy. BJOG 2003; 110:853-9.

http://www.ncbi.nlm.nih.gov/entrez/

query.fcgi?cmd $=$ Retrieve $\& \mathrm{db}=$ PubMed\&list_uids

$=14511969 \& \mathrm{dopt}=$ Abstract

Tench CM, McCarthy J, McCurdie I, et al. Fatigue in systemic lupus erythematosus: a randomized controlled trial of exercise. Rheumatology (Oxford) 2003;42:1050-4.

http://www.ncbi.nlm.nih.gov/entrez/

query.fcgi?cmd $=$ Retrieve $\& \mathrm{db}=$ PubMed\&list_uids

$=12730519 \&$ dopt $=$ Abstract

Terreehorst I, Hak E, Oosting AJ, et al. Evaluation of impermeable covers for bedding in patients with allergic rhinitis. N Engl J Med 2003;349:237-46.

http://www.ncbi.nlm.nih.gov/entrez/

query.fcgi? $\mathrm{cmd}=$ Retrieve $\& \mathrm{db}=$ PubMed\&list_uids

$=12867607 \& \mathrm{dopt}=$ Abstract

Toobert DJ, Glasgow RE, Strycker LA, et al. Biologic and quality-of-life outcomes from the Mediterranean Lifestyle Program: a randomized clinical trial. Diabetes Care 2003;26:2288-93. http://www.ncbi.nlm.nih.gov/entrez/

query.fcgi?cmd $=$ Retrieve\&db $=$ PubMed\&list_uids

$=12882850 \& \mathrm{dopt}=$ Abstract

TREC Collaborative Group. Rapid tranquillisation for agitated patients in emergency psychiatric rooms: a randomised trial of midazolam versus haloperidol plus promethazine. $B M J$ 2003;327:708-13.

http://www.ncbi.nlm.nih.gov/entrez/

query.fcgi?cmd $=$ Retrieve\&db $=$ PubMed\&list_uids

$=14512476 \& \mathrm{dopt}=$ Abstract

Tsang MW, Wong WK, Hung CS, et al. Human epidermal growth factor enhances healing of diabetic foot ulcers. Diabetes Care 2003;26:1856-61.

http://www.ncbi.nlm.nih.gov/entrez/

query.fcgi?cmd $=$ Retrieve $\& \mathrm{db}=$ PubMed\&list_uids

$=12766123 \& \mathrm{dopt}=$ Abstract
Verdon F, Burnand B, Stubi CL, et al. Iron supplementation for unexplained fatigue in non-anaemic women: double blind randomised placebo controlled trial. BMJ 2003;326:1124-7. http://www.ncbi.nlm.nih.gov/entrez/ query.fcgi?cmd $=$ Retrieve $\& \mathrm{db}=$ PubMed\&list_uids $=12763985 \& \mathrm{dopt}=$ Abstract

Verona E, Petrov D, Cserhati E, et al. Fluticasone propionate in asthma: a long term dose comparison study. Arch Dis Child 2003; 88:503-9.

http://www.ncbi.nlm.nih.gov/entrez/

query.fcgi?cmd = Retrieve\&db = PubMed\&list_uids

$=12765916 \& \mathrm{dopt}=$ Abstract

Voshaar RC, Gorgels WJ, Mol AJ, et al. Tapering off long-term benzodiazepine use with or without group cognitive-behavioural therapy: three-condition, randomised controlled trial. Br J Psychiatry 2003;182:498-504.

http://www.ncbi.nlm.nih.gov/entrez/

query.fcgi?cmd = Retrieve \&db = PubMed\&list_uids

$=12777340 \& \mathrm{dopt}=$ Abstract

Walker S, Monteil M, Phelan K, et al. Anti-IgE for chronic asthma. Cochrane Database Syst Rev 2003;(3):CD003559.

http://www.ncbi.nlm.nih.gov/entrez/

query.fcgi?cmd = Retrieve \&db = PubMed\&list uids

$=12917972 \& \mathrm{dopt}=$ Abstract

Wark PAB, Gibson PG, Wilson AJ. Azoles for allergic bronchopulmonary aspergillosis associated with asthma. Cochrane Database Syst Rev 2003;(3):CD001108.

http://www.ncbi.nlm.nih.gov/entrez/

query.fcgi? $\mathrm{cmd}=$ Retrieve $\& \mathrm{db}=$ PubMed\&list_uids

$=12917898 \& \mathrm{dopt}=$ Abstract

Wills CE, Holmes-Rovner M. Patient comprehension of information for shared treatment decision making: state of the art and future directions. Patient Educ Couns 2003;50:285-90.

http://www.ncbi.nlm.nih.gov/entrez/

query.fcgi?cmd $=$ Retrieve $\& \mathrm{db}=$ PubMed\&list_uids

$=12900101 \& \mathrm{dopt}=$ Abstract

Wilson SJ, Wells PS, Kovacs MJ, et al. Comparing the quality of oral anticoagulant management by anticoagulation clinics and by family physicians: a randomized controlled trial. CMAJ 2003; 169:293-8.

http://www.ncbi.nlm.nih.gov/entrez/

query.fcgi? $\mathrm{cmd}=$ Retrieve $\& \mathrm{db}=$ PubMed\&list_uids

$=12925422 \& \mathrm{dopt}=$ Abstract

Woodcock A, Forster L, Matthews E, et al. Control of exposure to mite allergen and allergen-impermeable bed covers for adults with asthma. $N$ Engl J Med 2003;349:225-36. http://www.ncbi.nlm.nih.gov/entrez/

query.fcgi? $\mathrm{cmd}=$ Retrieve $\& \mathrm{db}=$ PubMed\&list_uids $\mathrm{X}=12867606 \&$ dopt $=$ Abstract

Zandbergen AA, Baggen MG, Lamberts SW, et al. Effect of losartan on microalbuminuria in normotensive patients with type 2 diabetes mellitus. A randomized clinical trial. Ann Intern Med 2003;139:90-6.

http://www.ncbi.nlm.nih.gov/entrez/

query.fcgi?cmd $=$ Retrieve \&db $=$ PubMed\&list_uids

$=12859158 \& \mathrm{dopt}=$ Abstract

\section{ASSESSMENT (SCREENING OR DIAGNOSIS)}

Bonsu BK, Harper MB. Identifying febrile young infants with bacteremia: is the peripheral white blood cell count an accurate screen? Ann Emerg Med 2003;42:216-25. 
9 of 14

http://www.ncbi.nlm.nih.gov/entrez/

query.fcgi?cmd $=$ Retrieve\&db = PubMed\&list_uids

$=12883509 \& \mathrm{dopt}=$ Abstract

Hopstaken RM, Muris JW, Knottnerus JA, et al. Contributions of symptoms, signs, erythrocyte sedimentation rate, and Creactive protein to a diagnosis of pneumonia in acute lower respiratory tract infection. Br J Gen Pract 2003;53:358-64. http://www.ncbi.nlm.nih.gov/entrez/ query.fcgi?cmd $=$ Retrieve\&db $=$ PubMed\&list_uids $=12830562 \& \mathrm{dopt}=$ Abstract

Jackson JL, O'Malley PG, Kroenke K. Evaluation of acute knee pain in primary care. Ann Intern Med 2003;139:575-88. http://www.ncbi.nlm.nih.gov/entrez/

query.fcgi?cmd $=$ Retrieve\&db $=$ PubMed\&list_uids $=14530229 \& \mathrm{dopt}=$ Abstract

Lipton RB, Dodick D, Sadovsky R, et al. A self-administered screener for migraine in primary care: The ID Migraine(TM) validation study. Neurology 2003;61:375-82.

http://www.ncbi.nlm.nih.gov/entrez/

query.fcgi? $\mathrm{cmd}=$ Retrieve $\& \mathrm{db}=$ PubMed\&list uids

$=12913201 \& \mathrm{dopt}=$ Abstract

\section{PROGNOSIS}

Anderson P, Doyle LW. Neurobehavioral outcomes of schoolage children born extremely low birth weight or very preterm in the 1990s. JAMA 2003;289:3264-72.

http://www.ncbi.nlm.nih.gov/entrez/

query.fcgi? $\mathrm{cmd}=$ Retrieve $\& \mathrm{db}=$ PubMed\&list_uids

$=12824207 \& \mathrm{dopt}=$ Abstract

Critchley JA, Capewell S. Mortality risk reduction associated with smoking cessation in patients with coronary heart disease: a systematic review. JAMA 2003;290:86-97.

http://www.ncbi.nlm.nih.gov/entrez/

query.fcgi?cmd $=$ Retrieve \&db $=$ PubMed\&list uids

$=12837716 \& d o p t=$ Abstract

Di Carlo A, Lamassa M, Baldereschi M, et al. Sex differences in the clinical presentation, resource use, and 3-month outcome of acute stroke in Europe: data from a multicenter multinational hospital-based registry. Stroke 2003;34:1114-9. http://www.ncbi.nlm.nih.gov/entrez/

query.fcgi?cmd $=$ Retrieve \&db $=$ PubMed\&list_uids

$=12690218 \& \mathrm{dopt}=$ Abstract

Fowler RA, Lapinsky SE, Hallett D, et al. Critically ill patients with severe acute respiratory syndrome. JAMA 2003;290:367-73. http://www.ncbi.nlm.nih.gov/entrez/

query.fcgi?cmd $=$ Retrieve \&db $=$ PubMed\&list_uids

$=12865378 \& \mathrm{dopt}=$ Abstract

Garg AX, Suri RS, Barrowman N, et al. Long-term renal prognosis of diarrhea-associated hemolytic uremic syndrome: a systematic review, meta-analysis, and meta-regression. JAMA 2003;290:1360-70.

http://www.ncbi.nlm.nih.gov/entrez/

query.fcgi?cmd $=$ Retrieve\&db $=$ PubMed\&list_uids

$=12966129 \& \mathrm{dopt}=$ Abstract

Hallert E, Thyberg I, Hass U, et al. Comparison between women and men with recent onset rheumatoid arthritis of disease activity and functional ability over two years (the TIRA project). Ann Rheum Dis 2003;62:667-70.

http://www.ncbi.nlm.nih.gov/entrez/

query.fcgi?cmd $=$ Retrieve $\& \mathrm{db}=$ PubMed\&list_uids

$=12810432 \&$ dopt $=$ Abstract
Hawton K, Zahl D, Weatherall R. Suicide following deliberate self-harm: long-term follow-up of patients who presented to a general hospital. Br J Psychiatry 2003;182:537-42.

http://www.ncbi.nlm.nih.gov/entrez/

query.fcgi?cmd $=$ Retrieve $\& \mathrm{db}=$ PubMed\&list_uids

$=12777346 \&$ dopt $=$ Abstract

Hillen T, Coshall C, Tilling K, et al. Cause of stroke recurrence is multifactorial: patterns, risk factors, and outcomes of stroke recurrence in the South London Stroke Register. Stroke 2003;34:1457-63.

http://www.ncbi.nlm.nih.gov/entrez/

query.fcgi?cmd $=$ Retrieve $\& \mathrm{db}=$ PubMed\&list_uids

$=12750544 \&$ dopt $=$ Abstract

Hunt GM, Oakeshott P. Outcome in people with open spina bifida at age 35: prospective community based cohort study. BMJ 2003;326:1365-6.

http://www.ncbi.nlm.nih.gov/entrez/

query.fcgi?cmd $=$ Retrieve $\& \mathrm{db}=$ PubMed\&list_uids

$=12816823 \& \mathrm{dopt}=$ Abstract

Janssens HJ, van de Lisdonk EH, Bor H, et al. Gout, just a nasty event or a cardiovascular signal? A study from primary care. Fam Pract 2003;20:413-6.

http://www.ncbi.nlm.nih.gov/entrez/

query.fcgi?cmd $=$ Retrieve $\& \mathrm{db}=$ PubMed\&list_uids

$=12876112 \& \mathrm{dopt}=$ Abstract

Kiyohara Y, Kubo M, Kato I, et al. Ten-year prognosis of stroke and risk factors for death in a Japanese community: the Hisayama study. Stroke 2003;34:2343-7.

http://www.ncbi.nlm.nih.gov/entrez/

query.fcgi?cmd $=$ Retrieve $\& \mathrm{db}=$ PubMed\&list_uids

$=14500930 \& \mathrm{dopt}=$ Abstract

Lew TW, Kwek TK, Tai D, et al. Acute respiratory distress syndrome in critically ill patients with severe acute respiratory syndrome. JAMA 2003;290:374-80.

http://www.ncbi.nlm.nih.gov/entrez/

query.fcgi?cmd $=$ Retrieve $\& \mathrm{db}=$ PubMed\&list_uids

$=12865379 \& \mathrm{dopt}=$ Abstract

Pengel LH, Herbert RD, Maher CG, et al. Acute low back pain: systematic review of its prognosis. BMJ 2003;327:323-7.

http://www.ncbi.nlm.nih.gov/entrez/

query.fcgi?cmd $=$ Retrieve \&db $=$ PubMed\&list_uids

$=12907487 \& \mathrm{dopt}=$ Abstract

Roquer J, Campello AR, Gomis M. Sex differences in firstever acute stroke. Stroke 2003;34:1581-5.

http://www.ncbi.nlm.nih.gov/entrez/

query.fcgi?cmd $=$ Retrieve $\& \mathrm{db}=$ PubMed\&list_uids

$=12805490 \& \mathrm{dopt}=$ Abstract

Sejvar JJ, Haddad MB, Tierney BC, et al. Neurologic manifestations and outcome of West Nile virus infection. JAMA 2003;290:511-5.

http://www.ncbi.nlm.nih.gov/entrez/

query.fcgi?cmd = Retrieve\&db = PubMed\&list_uids

$=12876094 \& \mathrm{dopt}=$ Abstract

Vernino S, Brown RD Jr, Sejvar JJ, et al. Cause-specific mortality after first cerebral infarction: a population-based study. Stroke 2003;34:1828-32.

http://www.ncbi.nlm.nih.gov/entrez/

query.fcgi?cmd $=$ Retrieve $\& \mathrm{db}=$ PubMed\&list_uids

$=12855836 \& \mathrm{dopt}=$ Abstract

Zaw W, Gagnon R, da Silva O. The risks of adverse neonatal outcome among preterm small for gestational age infants 
10 of 14

according to neonatal versus fetal growth standards. Pediatrics 2003;111:1273-7.

http://www.ncbi.nlm.nih.gov/entrez/

query.fcgi?cmd $=$ Retrieve $\& \mathrm{db}=$ PubMed\&list_uids

$=12777541 \&$ dopt $=$ Abstract

\section{CAUSATION}

Bazzano LA, He J, Ogden LG, et al. Dietary fiber intake and reduced risk of coronary heart disease in US men and women: the National Health and Nutrition Examination Survey I Epidemiologic Follow-up Study. Arch Intern Med 2003; 163:1897-904.

http://www.ncbi.nlm.nih.gov/entrez/

query.fcgi?cmd $=$ Retrieve $\& \mathrm{db}=$ PubMed\&list_uids

$=12963562 \& \mathrm{dopt}=$ Abstract

Beral V. Breast cancer and hormone-replacement therapy in the Million Women Study. Lancet 2003;362:419-27. http://www.ncbi.nlm.nih.gov/entrez/ query.fcgi?cmd $=$ Retrieve $\& \mathrm{db}=$ PubMed\&list_uids $=12927427 \& \mathrm{dopt}=$ Abstract

Chae CU, Albert CM, Glynn RJ, et al. Mild renal insufficiency and risk of congestive heart failure in men and women $>$ or $=70$ years of age. Am J Cardiol 2003;92:682-6.

http://www.ncbi.nlm.nih.gov/entrez/

query.fcgi? $\mathrm{cmd}=$ Retrieve $\& \mathrm{db}=$ PubMed\&list_uids

$=12972106 \& \mathrm{dopt}=$ Abstract

Gill TM, Allore H, Guo Z. Restricted activity and functional decline among community-living older persons. Arch Intern Med 2003; 163:1317-22.

http://www.ncbi.nlm.nih.gov/entrez/

query.fcgi?cmd $=$ Retrieve \&db $=$ PubMed\&list_uids

$=12796067 \& \mathrm{dopt}=$ Abstract

Huizink AC, Robles de Medina PG, Mulder EJ, et al. Stress during pregnancy is associated with developmental outcome in infancy. $J$ Child Psychol Psychiatry 2003;44:810-8. http://www.ncbi.nlm.nih.gov/entrez/

query.fcgi? $\mathrm{cmd}=$ Retrieve $\& \mathrm{db}=$ PubMed\&list_uids

$=12959490 \& \mathrm{dopt}=$ Abstract

Kendler KS, Prescott CA, Myers J, et al. The structure of genetic and environmental risk factors for common psychiatric and substance use disorders in men and women. Arch Gen Psychiatry 2003;60:929-37.

http://www.ncbi.nlm.nih.gov/entrez/

query.fcgi?cmd $=$ Retrieve \&db = PubMed\&list_uids

$=12963675 \& \mathrm{dopt}=$ Abstract

Kiely DK, Flacker JM. The protective effect of social engagement on 1-year mortality in a long-stay nursing home population. J Clin Epidemiol 2003;56:472-8.

http://www.ncbi.nlm.nih.gov/entrez/

query.fcgi? $\mathrm{cmd}=$ Retrieve\&db = PubMed\&list_uids

$=12812822 \& \mathrm{dopt}=$ Abstract

Klatsky AL, Friedman GD, Armstrong MA, et al. Wine, liquor, beer, and mortality. Am J Epidemiol 2003;158:585-95.

http://www.ncbi.nlm.nih.gov/entrez/

query.fcgi?cmd $=$ Retrieve \&db $=$ PubMed\&list_uids

$=12965884 \& \mathrm{dopt}=$ Abstract

Lederle FA, Nelson DB, Joseph AM. Smokers' relative risk for aortic aneurysm compared with other smokingrelated diseases: a systematic review. J Vasc Surg 2003;38:329-34. http://www.ncbi.nlm.nih.gov/entrez/

query.fcgi?cmd $=$ Retrieve $\& \mathrm{db}=$ PubMed\&list_uids

$=12891116 \&$ dopt $=$ Abstract

Li DK, Liu L, Odouli R. Exposure to non-steroidal antiinflammatory drugs during pregnancy and risk of miscarriage: population based cohort study. BMJ 2003;327:368-72. http://www.ncbi.nlm.nih.gov/entrez/

query.fcgi?cmd $=$ Retrieve $\& \mathrm{db}=$ PubMed\&list_uids

$=12919986 \&$ dopt $=$ Abstract

Liang J, Bennett JM, Sugisawa H, et al. Gender differences in old age mortality: roles of health behavior and baseline health status. J Clin Epidemiol 2003;56:572-82.

http://www.ncbi.nlm.nih.gov/entrez/

query.fcgi?cmd $=$ Retrieve $\& \mathrm{db}=$ PubMed\&list_uids

$=12873653 \&$ dopt $=$ Abstract

Lipsky S, Holt VL, Easterling TR, et al. Impact of policereported intimate partner violence during pregnancy on birth outcomes. Obstet Gynecol 2003;102:557-64.

http://www.ncbi.nlm.nih.gov/entrez/

query.fcgi? $\mathrm{cmd}=$ Retrieve $\& \mathrm{db}=$ PubMed\&list_uids

$=12962943 \&$ dopt $=$ Abstract

Modin B. Born out of wedlock and never married-it breaks a man's heart. Soc Sci Med 2003;57:487-501.

http://www.ncbi.nlm.nih.gov/entrez/

query.fcgi?cmd $=$ Retrieve $\& \mathrm{db}=$ PubMed\&list_uids

$=12791491 \& \mathrm{dopt}=$ Abstract

Nybo H, Petersen HC, Gaist D, et al. Predictors of mortality in 2,249 nonagenarians-the Danish 1905-Cohort Survey. J Am Geriatr Soc 2003;51:1365-73.

http://www.ncbi.nlm.nih.gov/entrez/

query.fcgi?cmd $=$ Retrieve $\& \mathrm{db}=$ PubMed\&list_uids

$=14511155 \&$ dopt $=$ Abstract

Perk G, Stessman J, Ginsberg G, et al. Sex differences in the effect of heart rate on mortality in the elderly. J Am Geriatr Soc 2003;51:1260-4.

http://www.ncbi.nlm.nih.gov/entrez/

query.fcgi?cmd $=$ Retrieve \&db $=$ PubMed\&list uids

$=12919238 \&$ dopt $=$ Abstract

Qin P, Mortensen PB. The impact of parental status on the risk of completed suicide. Arch Gen Psychiatry 2003;60:797-802. http://www.ncbi.nlm.nih.gov/entrez/

query.fcgi? $\mathrm{cmd}=$ Retrieve $\& \mathrm{db}=$ PubMed\&list_uids

$=12912763 \& \mathrm{dopt}=$ Abstract

Runeson B, Asberg M. Family history of suicide among suicide victims. Am J Psychiatry 2003:160:1525-6.

http://www.ncbi.nlm.nih.gov/entrez/

query.fcgi?cmd $=$ Retrieve\&db $=$ PubMed\&list_uids

$=12900320 \& \mathrm{dopt}=$ Abstract

Rutledge T, Matthews K, Lui LY, et al. Social networks and marital status predict mortality in older women: prospective evidence from the Study of Osteoporotic Fractures (SOF). Psychosom Med 2003;65:688-94.

http://www.ncbi.nlm.nih.gov/entrez/

query.fcgi?cmd = Retrieve\&db = PubMed\&list uids

$=12883123 \&$ dopt $=$ Abstract

Smith GC, Pell JP, Dobbie R. Interpregnancy interval and risk of preterm birth and neonatal death: retrospective cohort study. BMJ 2003;327:313-8.

http://www.ncbi.nlm.nih.gov/entrez/

query.fcgi?cmd $=$ Retrieve\&db $=$ PubMed\&list_uids

$=12907483 \& \mathrm{dopt}=$ Abstract 
Stephansson O, Dickman PW, Cnattingius S. The influence of interpregnancy interval on the subsequent risk of stillbirth and early neonatal death. Obstet Gynecol 2003;102:101-8.

http://www.ncbi.nlm.nih.gov/entrez/

query.fcgi?cmd $=$ Retrieve \&db $=$ PubMed\&list_uids

$=12850614 \& \mathrm{dopt}=$ Abstract

Vessey M, Painter R, Yeates D. Mortality in relation to oral contraceptive use and cigarette smoking. Lancet 2003;362:185-91. http://www.ncbi.nlm.nih.gov/entrez/

query.fcgi?cmd $=$ Retrieve $\&$ db $=$ PubMed\&list_uids

$=12885478 \& \mathrm{dopt}=$ Abstract

Weverling-Rijnsburger AW, Jonkers IJ, van Exel E, et al. High-density vs low-density lipoprotein cholesterol as the risk factor for coronary artery disease and stroke in old age. Arch Intern Med 2003;163:1549-54.

http://www.ncbi.nlm.nih.gov/entrez/

query.fcgi?cmd $=$ Retrieve $\& \mathrm{db}=$ PubMed\&list_uids

$=12860577 \& \mathrm{dopt}=$ Abstract

\section{QUALITY IMPROVEMENT}

Ansari M, Shlipak MG, Heidenreich PA, et al. Improving guideline adherence: a randomized trial evaluating strategies to increase beta-blocker use in heart failure. Circulation 2003;107:2799-804.

http://www.ncbi.nlm.nih.gov/entrez/

query.fcgi?cmd $=$ Retrieve $\& \mathrm{db}=$ PubMed\&list_uids

$=12756157 \& \mathrm{dopt}=$ Abstract

Bonner AB, Monroe KW, Talley LI, et al. Impact of the rapid diagnosis of influenza on physician decision-making and patient management in the pediatric emergency department: results of a randomized, prospective, controlled trial. Pediatrics 2003; 112:363-7.

http://www.ncbi.nlm.nih.gov/entrez/

query.fcgi?cmd $=$ Retrieve\&db $=$ PubMed\&list_uids

$=12897288 \& \mathrm{dopt}=$ Abstract

Bordley WC, Travers D, Scanlon P, et al. Office preparedness for pediatric emergencies: a randomized, controlled trial of an office-based training program. Pediatrics 2003;112:291-5.

http://www.ncbi.nlm.nih.gov/entrez/

query.fcgi? $\mathrm{cmd}=$ Retrieve $\& \mathrm{db}=$ PubMed\&list_uids

$=12897276 \& \mathrm{dopt}=$ Abstract

Currell R, Urquhart C. Nursing record systems: effects on nursing practice and health care outcomes. Cochrane Database Syst Rev 2003;(3):CD002099.

http://www.ncbi.nlm.nih.gov/entrez/

query.fcgi?cmd $=$ Retrieve $\& \mathrm{db}=$ PubMed\&list_uids

$=12917920 \&$ dopt $=$ Abstract

Daly S, Campbell DA, Cameron PA. Short-stay units and observation medicine: a systematic review. Med J Aust 2003;178:559-63.

http://www.ncbi.nlm.nih.gov/entrez/

query.fcgi?cmd $=$ Retrieve\&db = PubMed\&list_uids

$=12765504 \& \mathrm{dopt}=$ Abstract

Hicks C, Spurgeon P, Barwell F. Changing Childbirth: a pilot project. $J$ Adv Nurs 2003;42:617-28.

http://www.ncbi.nlm.nih.gov/entrez/

query.fcgi? $\mathrm{cmd}=$ Retrieve $\& \mathrm{db}=$ PubMed\&list_uids

$=12787235 \& \mathrm{dopt}=$ Abstract

Jamtvedt G, Young JM, Kristoffersen DT, et al. Audit and feedback: effects on professional practice and health care outcomes. Cochrane Database Syst Rev 2003;(3):CD000259. http://www.ncbi.nlm.nih.gov/entrez/

query.fcgi? $\mathrm{cmd}=$ Retrieve $\& \mathrm{db}=$ PubMed\&list_uids

$=12917891 \& \mathrm{dopt}=$ Abstract

Kaushal R, Shojania KG, Bates DW. Effects of computerized physician order entry and clinical decision support systems on medication safety: a systematic review. Arch Intern Med 2003;163:1409-16.

http://www.ncbi.nlm.nih.gov/entrez/

query.fcgi?cmd $=$ Retrieve $\& \mathrm{db}=$ PubMed\&list_uids

$=12824090 \& \mathrm{dopt}=$ Abstract

van Dam HA, van der Horst F, van den Borne B, et al. Provider-patient interaction in diabetes care: effects on patient self-care and outcomes. A systematic review. Patient Educ Couns 2003;51:17-28.

http://www.ncbi.nlm.nih.gov/entrez/

query.fcgi?cmd $=$ Retrieve $\& \mathrm{db}=$ PubMed\&list_uids

$=12915276 \&$ dopt $=$ Abstract

Webster J, Pritchard MA. Gowning by attendants and visitors in newborn nurseries for prevention of neonatal morbidity and mortality. Cochrane Database Syst Rev 2003;(3):CD003670. http://www.ncbi.nlm.nih.gov/entrez/

query.fcgi?cmd $=$ Retrieve $\& \mathrm{db}=$ PubMed\&list_uids

$=12917980 \& \mathrm{dopt}=$ Abstract

\section{CLINICAL PREDICTION GUIDE}

Davis EG, MacKenzie EJ, Sacco WJ, et al. A new "TRISS-like" probability of survival model for intubated trauma patients. $J$ Trauma 2003;55:53-61.

http://www.ncbi.nlm.nih.gov/entrez/

query.fcgi?cmd $=$ Retrieve \&db = PubMed\&list_uids

$=12855881 \& \mathrm{dopt}=$ Abstract

De Jonge P, Bauer I, Huyse FJ, et al. Medical inpatients at risk of extended hospital stay and poor discharge health status: detection with COMPRI and INTERMED. Psychosom Med 2003;65:534-41.

http://www.ncbi.nlm.nih.gov/entrez/

query.fcgi?cmd $=$ Retrieve $\& d b=$ PubMed\&list_uids

$=12883102 \& \mathrm{dopt}=$ Abstract

Hitsman B, Borrelli B, McChargue DE, et al. History of depression and smoking cessation outcome: a meta-analysis. J Consult Clin Psychol 2003;71:657-63.

http://www.ncbi.nlm.nih.gov/entrez/

query.fcgi?cmd $=$ Retrieve $\& \mathrm{db}=$ PubMed\&list_uids

$=12924670 \& \mathrm{dopt}=$ Abstract

Kline JA, Hernandez-Nino J, Newgard CD, et al. Use of pulse oximetry to predict in-hospital complications in normotensive patients with pulmonary embolism. Am J Med 2003;115:203-8. http://www.ncbi.nlm.nih.gov/entrez/

query.fcgi? $\mathrm{cmd}=$ Retrieve $\& \mathrm{db}=$ PubMed\&list_uids

$=12935827 \& \mathrm{dopt}=$ Abstract

\section{ECONOMICS}

O'Brien JF, Grace PA, Perry IJ, et al. Randomized clinical trial and economic analysis of four-layer compression bandaging for venous ulcers. Br J Surg 2003;90:794-8.

http://www.ncbi.nlm.nih.gov/entrez/

query.fcgi?cmd = Retrieve\&db = PubMed\&list_uids

$=12854102 \& \mathrm{dopt}=$ Abstract

\section{QUALITATIVE}

Aitken LM. Critical care nurses' use of decision-making strategies. J Clin Nurs 2003;12:476-83. 
12 of 14

http://www.ncbi.nlm.nih.gov/entrez/

query.fcgi?cmd $=$ Retrieve $\& \mathrm{db}=$ PubMed\&list_uids

$=12790860 \& \mathrm{dopt}=$ Abstract

Allotey P, Reidpath D, Kouamé A, et al. The DALY, context and the determinants of the severity of disease: an exploratory comparison of paraplegia in Australia and Cameroon. Soc Sci Med 2003;57:949-58.

http://www.ncbi.nlm.nih.gov/entrez/

query.fcgi?cmd $=$ Retrieve \&db $=$ PubMed\&list_uids

$=12850119 \& \mathrm{dopt}=$ Abstract

Banister EM, Jakubec SL, Stein JA. "Like, what am I supposed to do?": adolescent girls' health concerns in their dating relationships. Can J Nurs Res 2003;35:16-33.

http://www.ncbi.nlm.nih.gov/entrez/

query.fcgi?cmd $=$ Retrieve $\& \mathrm{db}=$ PubMed\&list_uids

$=12908195 \& \mathrm{dopt}=$ Abstract

Bhavnani V, Clarke A. Women awaiting hysterectomy: a qualitative study of issues involved in decisions about oophorectomy. BJOG 2003;110:168-74.

http://www.ncbi.nlm.nih.gov/entrez/

query.fcgi?cmd $=$ Retrieve $\& \mathrm{db}=$ PubMed\&list_uids

$=12618161 \& \mathrm{dopt}=$ Abstract

Blake DR, Kearney $\mathrm{MH}$, Oakes JM, et al. Improving participation in chlamydia screening programs: perspectives of high-risk youth. Arch Pediatr Adolesc Med 2003;157:523-9. http://www.ncbi.nlm.nih.gov/entrez/

query.fcgi?cmd $=$ Retrieve $\& \mathrm{db}=$ PubMed\&list_uids

$=12796231 \& \mathrm{dopt}=$ Abstract

Brown P, Mayer B, Zavestoski S, et al. The health politics of asthma: environmental justice and collective illness experience in the United States. Soc Sci Med 2003;57:453-64.

http://www.ncbi.nlm.nih.gov/entrez/

query.fcgi?cmd $=$ Retrieve\&db $=$ PubMed\&list_uids

$=12791488 \& \mathrm{dopt}=$ Abstract

Bowles KH, Foust JB, Naylor MD. Hospital discharge referral decision making: a multidisciplinary perspective. Appl Nurs Res 2003;16:134-43.

http://www.ncbi.nlm.nih.gov/entrez/

query.fcgi?cmd $=$ Retrieve $\& \mathrm{db}=$ PubMed\&list_uids

$=12931327 \& \mathrm{dopt}=$ Abstract

Callery P, Milnes L, Chrissie V, et al. Qualitative study of young people's and parents' beliefs about childhood asthma. Br J Gen Pract 2003;53:185-90.

Cassells C, Watt E. The impact of incontinence on older spousal caregivers. J Adv Nurs 2003;42:607-16.

http://www.ncbi.nlm.nih.gov/entrez/

query.fcgi?cmd $=$ Retrieve $\& \mathrm{db}=$ PubMed\&list_uids

$=12787234 \& \mathrm{dopt}=$ Abstract

Cescutti-Butler L, Galvin K. Parents' perceptions of staff competency in a neonatal intensive care unit. J Clin Nurs 2003; 12:752-61.

http://www.ncbi.nlm.nih.gov/entrez/

query.fcgi? $\mathrm{cmd}=$ Retrieve $\& \mathrm{db}=$ PubMed\&list_uids

$=12919222 \& \mathrm{dopt}=$ Abstract

Chapman RR. Endangering safe motherhood in Mozambique: prenatal care as pregnancy risk. Soc Sci Med 2003; 57:355-74.

http://www.ncbi.nlm.nih.gov/entrez/

query.fcgi?cmd $=$ Retrieve $\& \mathrm{db}=$ PubMed\&list_uids

$=12765714 \& \mathrm{dopt}=$ Abstract
Clarke JN, Fletcher P. Communication issues faced by parents who have a child diagnosed with cancer. $J$ Pediatr Oncol Nurs 2003;20:175-91.

http://www.ncbi.nlm.nih.gov/entrez/

query.fcgi?cmd $=$ Retrieve $\& \mathrm{db}=$ PubMed\&list_uids

$=14567565 \&$ dopt $=$ Abstract

Cooper H, Booth K, Gill G. Using combined research methods for exploring diabetes patient education. Patient Educ Couns 2003; 51:45-52.

http://www.ncbi.nlm.nih.gov/entrez/

query.fcgi?cmd $=$ Retrieve $\& \mathrm{db}=$ PubMed\&list_uids

$=12915279 \& \mathrm{dopt}=$ Abstract

Copeland L. An exploration of the problems faced by young women living in disadvantaged circumstances if they want to give up smoking: can more be done at general practice level? Fam Pract 2003;20:393-400.

http://www.ncbi.nlm.nih.gov/entrez/

query.fcgi?cmd $=$ Retrieve $\& d b=$ PubMed\&list_uids

$=12876109 \& \mathrm{dopt}=$ Abstract

Curtis V, Biran A, Deverell K, et al. Hygiene in the home: relating bugs and behaviour. Soc Sci Med 2003;57:657-72. http://www.ncbi.nlm.nih.gov/entrez/

query.fcgi?cmd = Retrieve \&db = PubMed\&list_uids

$=12821014 \& \mathrm{dopt}=$ Abstract

Dolan KA, Davis PW. Nuances and shifts in lesbian women's constructions of STI and HIV vulnerability. Soc Sci Med 2003;57:25-38

http://www.ncbi.nlm.nih.gov/entrez/

query.fcgi?cmd $=$ Retrieve $\& \mathrm{db}=$ PubMed\&list_uids

$=12753814 \& \mathrm{dopt}=$ Abstract

Donohue RK. Nurse practitioner-client interaction as resource exchange in a women's health clinic: an exploratory study. J Clin Nurs 2003;12:717-25.

http://www.ncbi.nlm.nih.gov/entrez/

query.fcgi?cmd $=$ Retrieve $\& \mathrm{db}=$ PubMed\&list_uids

$=12919218 \& \mathrm{dopt}=$ Abstract

Edvardsson JD, Sandman PO, Rasmussen BH. Meanings of giving touch in the care of older patients: becoming a valuable person and professional. J Clin Nurs 2003;12:601-9. http://www.ncbi.nlm.nih.gov/entrez/

query.fcgi?cmd $=$ Retrieve $\& \mathrm{db}=$ PubMed\&list_uids

$=12790874 \& \mathrm{dopt}=$ Abstract

Evangelista LS, Doering L, Dracup K. Meaning and life purpose: the perspectives of post-transplant women. Heart Lung 2003;32:250-7.

http://www.ncbi.nlm.nih.gov/entrez/

query.fcgi? $\mathrm{cmd}=$ Retrieve $\& \mathrm{db}=$ PubMed\&list_uids

$=12891165 \& \mathrm{dopt}=$ Abstract

Finnbogadóttir H, Crang Svalenius E, Persson EK. Expectant first-time fathers' experiences of pregnancy. Midwifery 2003; 19:96-105.

http://www.ncbi.nlm.nih.gov/entrez/

query.fcgi?cmd $=$ Retrieve $\& d b=$ PubMed\&list_uids

$=12809629 \& \mathrm{dopt}=$ Abstract

Free C, Green J, Bhavnani V, et al. Bilingual young people's experiences of interpreting in primary care: a qualitative study. Br J Gen Pract 2003;53:530-5.

Freedman TG. Prescriptions for health providers: from cancer patients. Cancer Nurs 2003;26:323-30. 
http://www.ncbi.nlm.nih.gov/entrez/

query.fcgi? $\mathrm{cmd}=$ Retrieve $\& \mathrm{db}=$ PubMed\&list_uids

$=12886123 \& \mathrm{dopt}=$ Abstract

Gage-Rancoeur DM, Purden MA. Daughters of cardiac patients: the process of caregiving. Can J Nurs Res 2003;35:90-105.

http://www.ncbi.nlm.nih.gov/entrez/

query.fcgi?cmd $=$ Retrieve $\& \mathrm{db}=$ PubMed\&list_uids

$=12908199 \& \mathrm{dopt}=$ Abstract

Gagliardi BA. The experience of sexuality for individuals living with multiple sclerosis. J Clin Nurs 2003;12:571-8.

http://www.ncbi.nlm.nih.gov/entrez/

query.fcgi? $\mathrm{cmd}=$ Retrieve $\& \mathrm{db}=$ PubMed\&list_uids

$=12790871 \& \mathrm{dopt}=$ Abstract

Gallagher TH, Waterman AD, Ebers AG, et al. Patients' and physicians' attitudes regarding the disclosure of medical errors. JAMA 2003;289:1001-7.

http://www.ncbi.nlm.nih.gov/entrez/

query.fcgi?cmd $=$ Retrieve $\& \mathrm{db}=$ PubMed\&list_uids

$=12597752 \& \mathrm{dopt}=$ Abstract

Gooberman-Hill R, Ayis S, Ebrahim S. Understanding longstanding illness among older people. Soc Sci Med 2003:56:2555-64.

http://www.ncbi.nlm.nih.gov/entrez/

query.fcgi?cmd $=$ Retrieve $\& \mathrm{db}=$ PubMed\&list_uids

$=12742617 \& \mathrm{dopt}=$ Abstract

Greaves CJ, Brown P, Terry RT, et al. Converting to insulin in primary care: an exploration of the needs of practice nurses. $J$ Adv Nurs 2003;42:487-96.

http://www.ncbi.nlm.nih.gov/entrez/

query.fcgi?cmd $=$ Retrieve $\& \mathrm{db}=$ PubMed\&list_uids

$=12752869 \& \mathrm{dopt}=$ Abstract

Haglund K. Sexually abstinent African American adolescent females' descriptions of abstinence. $J$ Nurs Scholarsh 2003;35:231-6.

http://www.ncbi.nlm.nih.gov/entrez/

query.fcgi?cmd $=$ Retrieve $\& \mathrm{db}=$ PubMed\&list_uids

$=14562490 \& \mathrm{dopt}=$ Abstract

Hamilton JB, Sandelowski M. Living the golden rule: reciprocal exchanges among African Americans with cancer. Qual Health Res 2003;13:656-74.

http://www.ncbi.nlm.nih.gov/entrez/

query.fcgi?cmd = Retrieve \&db = PubMed\&list_uids

$=12756686 \& \mathrm{dopt}=$ Abstract

Harrison MJ, Kushner KE, Benzies $\mathrm{K}$, et al. Women's satisfaction with their involvement in health care decisions during a high-risk pregnancy. Birth 2003;30:109-15.

http://www.ncbi.nlm.nih.gov/entrez/

query.fcgi?cmd $=$ Retrieve $\& \mathrm{db}=$ PubMed\&list_uids

$=12752168 \& \mathrm{dopt}=$ Abstract

Hayward C, Madill A. The meanings of organ donation: Muslims of Pakistani origin and white English nationals living in North England. Soc Sci Med 2003;57:389-401.

http://www.ncbi.nlm.nih.gov/entrez/

query.fcgi? $\mathrm{cmd}=$ Retrieve $\& \mathrm{db}=$ PubMed\&list_uids

$=12791483 \& \mathrm{dopt}=$ Abstract

Henderson PD, Gore SV, Davis BL, et al. African American women coping with breast cancer: a qualitative analysis. Oncol Nurs Forum 2003;30:641-7.

http://www.ncbi.nlm.nih.gov/entrez/

query.fcgi?cmd $=$ Retrieve $\& \mathrm{db}=$ PubMed\&list_uids

$=12861324 \& \mathrm{dopt}=$ Abstract
13 of 14

Henderson S. Power imbalance between nurses and patients: a potential inhibitor of partnership in care. $J$ Clin Nurs 2003; 12:501-8.

http://www.ncbi.nlm.nih.gov/entrez/

query.fcgi?cmd $=$ Retrieve\&db $=$ PubMed\&list_uids

$=12790863 \& \mathrm{dopt}=$ Abstract

Holmström I, Halford C, Rosenqvist U. Swedish health care professionals' diverse understandings of diabetes care. Patient Educ Couns 2003;51:53-8.

http://www.ncbi.nlm.nih.gov/entrez/

query.fcgi?cmd $=$ Retrieve $\& \mathrm{db}=$ PubMed\&list_uids

$=12915280 \& \mathrm{dopt}=$ Abstract

Jacelon CS. The dignity of elders in an acute care hospital. Qual Health Res 2003;13:543-56.

http://www.ncbi.nlm.nih.gov/entrez/

query.fcgi? $\mathrm{cmd}=$ Retrieve $\& \mathrm{db}=$ PubMed\&list_uids

$=12703415 \& \mathrm{dopt}=$ Abstract

Jackson K, Ternestedt BM, Schollin J. From alienation to familiarity: experiences of mothers and fathers of preterm infants. $J$ Adv Nurs 2003;43:120-9.

http://www.ncbi.nlm.nih.gov/entrez/

query.fcgi?cmd $=$ Retrieve $\& \mathrm{db}=$ PubMed\&list_uids

$=12834369 \& \mathrm{dopt}=$ Abstract

Joachim G, Acorn S. Life with a rare chronic disease: the scleroderma experience. $J$ Adv Nurs 2003;42:598-606.

http://www.ncbi.nlm.nih.gov/entrez/

query.fcgi .cmd $=$ Retrieve $\& \mathrm{db}=$ PubMed\&list_uids

$=12787233 \& \mathrm{dopt}=$ Abstract

Kim SJ, Kang KA. Meaning of life for adolescents with a physical disability in Korea. $J$ Adv Nurs 2003;43:145-55.

http://www.ncbi.nlm.nih.gov/entrez/

query.fcgi? $\mathrm{cmd}=$ Retrieve $\& \mathrm{db}=$ PubMed\&list_uids

$=12834372 \& \mathrm{dopt}=$ Abstract

Kyngäs H. Patient education: perspective of adolescents with a chronic disease. J Clin Nurs 2003;12:744-51.

http://www.ncbi.nlm.nih.gov/entrez/

query.fcgi?cmd $=$ Retrieve $\& \mathrm{db}=$ PubMed\&list_uids

$=12919221 \& \mathrm{dopt}=$ Abstract

Larsson M, Hedelin B, Athlin E. Lived experiences of eating problems for patients with head and neck cancer during radiotherapy. J Clin Nurs 2003;12:562-70.

http://www.ncbi.nlm.nih.gov/entrez/

query.fcgi? $\mathrm{cmd}=$ Retrieve $\& \mathrm{db}=$ PubMed\&list_uids

$=12790870 \& \mathrm{dopt}=$ Abstract

Lock LR, Gibb HJ. The power of place. Midwifery 2003;19:1329.

http://www.ncbi.nlm.nih.gov/entrez/

query.fcgi?cmd $=$ Retrieve $\& \mathrm{db}=$ PubMed\&list_uids

$=12809633 \& \mathrm{dopt}=$ Abstract

Lundqvist A, Nilstun T, Dykes AK. Both empowered and powerless: mothers' experiences of professional care when their newborn dies. Birth 2002;29:192-9.

http://www.ncbi.nlm.nih.gov/entrez/

query.fcgi?cmd = Retrieve\&db = PubMed\&list_uids

$=12153650 \& \mathrm{dopt}=$ Abstract

Lymer UB, Richt B, Isaksson B. Health care workers' action strategies in situations that involve a risk of blood exposure. $J$ Clin Nurs 2003;12:660-7.

http://www.ncbi.nlm.nih.gov/entrez/

query.fcgi?cmd $=$ Retrieve $\& \mathrm{db}=$ PubMed\&list_uids

$=12919212 \& \mathrm{dopt}=$ Abstract 
14 of 14

Majomi P, Brown B, Crawford P. Sacrificing the personal to the professional: community mental health nurses. $J$ Adv Nurs 2003;42:527-38.

http://www.ncbi.nlm.nih.gov/entrez/

query.fcgi?cmd $=$ Retrieve \&db = PubMed\&list_uids

$=12752873 \& \mathrm{dopt}=$ Abstract

Marshall MN, Mannion R, Nelson E, et al. Managing change in the culture of general practice: qualitative case studies in primary care trusts. BMJ 2003;327:599-602.

http://www.ncbi.nlm.nih.gov/entrez/

query.fcgi?cmd $=$ Retrieve $\& \mathrm{db}=$ PubMed\&list_uids

$=12969929 \& \mathrm{dopt}=$ Abstract

Martinson IM, Yee KH. Parental involvement in restoring the health of a child with cancer in Hong Kong. $J$ Pediatr Oncol Nurs 2003;20:233-44.

McCarthy MC. Detecting acute confusion in older adults: comparing clinical reasoning of nurses working in acute, long-term, and community health care environments. Res Nurs Health 2003;26:203-12.

http://www.ncbi.nlm.nih.gov/entrez/

query.fcgi?cmd $=$ Retrieve $\& \mathrm{db}=$ PubMed\&list_uids

$=12754728 \& \mathrm{dopt}=$ Abstract

Melanson PM, Downe-Wamboldt B. Confronting life with rheumatoid arthritis. J Adv Nurs 2003;42:125-33.

http://www.ncbi.nlm.nih.gov/entrez/

query.fcgi?cmd $=$ Retrieve\&db $=$ PubMed\&list_uids

$=12670381 \& \mathrm{dopt}=$ Abstract

Moffatt S, Pless-Mulloli T. "It wasn't the plague we expected." Parents' perceptions of the health and environmental impact of opencast coal mining. Soc Sci Med 2003;57:437-51.

http://www.ncbi.nlm.nih.gov/entrez/

query.fcgi?cmd $=$ Retrieve\&db $=$ PubMed\&list_uids

$=12791487 \& \mathrm{dopt}=$ Abstract

Mohr WK. The substance of a support group. West J Nurs Res 2003;25:676-92.

http://www.ncbi.nlm.nih.gov/entrez/

query.fcgi?cmd $=$ Retrieve $\& \mathrm{db}=$ PubMed\&list_uids

$=14528617 \& \mathrm{dopt}=$ Abstract

Mok E, Chan F, Chan V, et al. Family experience caring for terminally ill patients with cancer in Hong Kong. Cancer Nurs 2003;26:267-75.

http://www.ncbi.nlm.nih.gov/entrez/

query.fcgi?cmd $=$ Retrieve $\& \mathrm{db}=$ PubMed\&list_uids

$=12886117 \& \mathrm{dopt}=$ Abstract

Moore LW, Miller M. Older men's experiences of living with severe visual impairment. $J$ Adv Nurs 2003;43:10-8.

http://www.ncbi.nlm.nih.gov/entrez/

query.fcgi?cmd $=$ Retrieve $\& \mathrm{db}=$ PubMed\&list_uids

$=12801392 \& \mathrm{dopt}=$ Abstract

Nurse J, Woodcock P, Ormsby J. Influence of environmental factors on mental health within prisons: focus group study. BMJ 2003;327:480-4.

http://www.ncbi.nlm.nih.gov/entrez/

query.fcgi?cmd $=$ Retrieve \&db = PubMed\&list_uids

$=12946970 \& \mathrm{dopt}=$ Abstract

Odegaard G, Lindbladh E, Hovelius B. Children who suffer from headaches - a narrative of insecurity in school and family. Br J Gen Pract 2003;53:210-5.

Perry L, McLaren S. Eating difficulties after stroke. $J$ Adv Nurs 2003;43:360-9. http://www.ncbi.nlm.nih.gov/entrez/

query.fcgi?cmd $=$ Retrieve $\& \mathrm{db}=$ PubMed\&list_uids

$=12887354 \& \mathrm{dopt}=$ Abstract

Popay J, Thomas C, Williams G, et al. A proper place to live: health inequalities, agency and the normative dimensions of space. Soc Sci Med 2003;57:55-69.

http://www.ncbi.nlm.nih.gov/entrez/

query.fcgi?cmd $=$ Retrieve $\& \mathrm{db}=$ PubMed\&list_uids

$=12753816 \& d o p t=$ Abstract

Rapport F. Exploring the beliefs and experiences of potential egg share donors. J Adv Nurs 2003;43:28-42.

http://www.ncbi.nlm.nih.gov/entrez/

query.fcgi?cmd = Retrieve\&db = PubMed\&list_uids

$=12801394 \& \mathrm{dopt}=$ Abstract

Rew L. A theory of taking care of oneself grounded in experiences of homeless youth. Nurs Res 2003;52:234-41.

http://www.ncbi.nlm.nih.gov/entrez/

query.fcgi?cmd $=$ Retrieve $\& d b=$ PubMed\&list_uids

$=12867780 \& \mathrm{dopt}=$ Abstract

Rhodes T, Mikhailova L, Sarang A, et al. Situational factors influencing drug injecting, risk reduction and syringe exchange in Togliatti City, Russian Federation: a qualitative study of micro risk environment. Soc Sci Med 2003;57:39-54. http://www.ncbi.nlm.nih.gov/entrez/

query.fcgi?cmd $=$ Retrieve $\& \mathrm{db}=$ PubMed\&list_uids

$=12753815 \& \mathrm{dopt}=$ Abstract

Simmons B, Lanuza D, Fonteyn M, et al. Clinical reasoning in experienced nurses. West J Nurs Res 2003;25:701-19.

http://www.ncbi.nlm.nih.gov/entrez/

query.fcgi? $\mathrm{cmd}=$ Retrieve $\& \mathrm{db}=$ PubMed\&list_uids

$=14528618 \& \mathrm{dopt}=$ Abstract

Simpson PB. Family beliefs about diet and traditional Chinese medicine for Hong Kong women with breast cancer. Oncol Nurs Forum 2003;30:834-40.

http://www.ncbi.nlm.nih.gov/entrez/

query.fcgi?cmd $=$ Retrieve $\& \mathrm{db}=$ PubMed\&list_uids

$=12949596 \& \mathrm{dopt}=$ Abstract

Stevenson FA, Britten N, Barry CA, et al. Self-treatment and its discussion in medical consultations: how is medical pluralism managed in practice? Soc Sci Med 2003;57:513-27. http://www.ncbi.nlm.nih.gov/entrez/

query.fcgi?cmd $=$ Retrieve $\& \mathrm{db}=$ PubMed\&list_uids

$=12791493 \&$ dopt $=$ Abstract

Stewart JL. "Getting used to it": children finding the ordinary and routine in the uncertain context of cancer. Qual Health Res 2003; 13:394-407.

http://www.ncbi.nlm.nih.gov/entrez/

query.fcgi?cmd $=$ Retrieve $\& \mathrm{db}=$ PubMed\&list_uids

$=12669339 \& \mathrm{dopt}=$ Abstract

Taylor EJ. Nurses caring for the spirit: patients with cancer and family caregiver expectations. Oncol Nurs Forum 2003;30:585-90.

http://www.ncbi.nlm.nih.gov/entrez/

query.fcgi?cmd = Retrieve\&db = PubMed\&list_uids

$=12861319 \& \mathrm{dopt}=$ Abstract

Tarrant C, Windridge K, Boulton M, et al. Qualitative study of the meaning of personal care in general practice. $B M J$ 2003;326:1310-7.

http://www.ncbi.nlm.nih.gov/entrez/

query.fcgi?cmd $=$ Retrieve $\& d b=$ PubMed\&list_uids

$=12805168 \& \mathrm{dopt}=$ Abstract 